\title{
THE ASSOCIATED ASKEY-WILSON POLYNOMIALS
}

\author{
MOURAD E. H. ISMAIL AND MIZAN RAHMAN
}

\begin{abstract}
We derive some contiguous relations for very well-poised ${ }_{8} \phi_{7}$ series and use them to construct two linearly independent solutions of the three-term recurrence relation of the associated Askey-Wilson polynomials. We then use these solutions to find explicit representations of two families of associated Askey-Wilson polynomials. We identify the corresponding continued fractions as quotients of two very well-poised ${ }_{8} \phi_{7}$ series and find the weight functions.
\end{abstract}

\section{INTRODUCTION}

Throughout this work we shall assume that $q$ is a fixed number in $(0,1)$. A $q$-shifted factorial $(a)_{n}$ is defined by

$$
(a)_{0}=1,(a)_{n}=(1-a)(1-a q) \cdots\left(1-a q^{n-1}\right), \quad n=1,2, \ldots .
$$

Following Gasper and Rahman [13] we shall use the notation

$$
\left(a_{1}, a_{2}, \ldots, a_{k}\right)_{n}=\prod_{j=1}^{k}\left(a_{j}\right)_{n}, \quad n=0,1, \ldots
$$

The value $n=\infty$ is allowed in (1.1) and (1.2). Normally the dependence on the base $q$ is indicated by using $(a ; q)_{n}$ and $\left(a_{1}, \ldots, a_{k} ; q\right)_{n}$ instead of $(a)_{n}$ and $\left(a_{1}, \ldots, a_{k}\right)_{n}$, respectively. However, since we will be using only one base throughout the paper we decided to delete its explicit display.

A basic hypergeometric series with $r+1$ numerator and $r$ denominator parameters is defined by

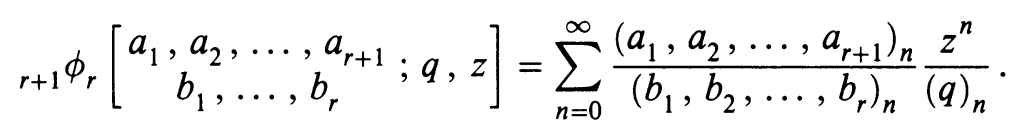

The Askey-Wilson polynomials [7] are the ${ }_{4} \varphi_{3}$ polynomials

$$
\begin{array}{r}
p_{n}(x ; a, b, c, d \mid q)={ }_{4} \phi_{3}\left[\begin{array}{c}
q^{-n}, a b c d q^{n-1}, a z, a / z \\
a b, a c, a d
\end{array} ; q, q\right], \\
x=\left(z+z^{-1}\right) / 2,|z| \leq 1 .
\end{array}
$$

Received by the editors May 8, 1989 and, in revised form, September 8, 1989.

1980 Mathematics Subject Classification (1985 Revision). Primary 33A65; Secondary 42C05.

The research of the first author was partially supported by NSF grant DMS 8714630 .

The research of the second author was partially supported by NSERC (Canada) grant A6197. 
Note that the product of the denominator parameters in this ${ }_{4} \phi_{3}$ series is $q$ times the product of the numerator parameters, and the argument of the function is $q$. Basic hypergeometric series having this property are called balanced [13] (Saalschützian in [9]). It is easy to see that if $x=\cos \theta$ in (1.4), then $z=e^{-i \theta}$. The Askey-Wilson polynomials are the most general family of orthogonal polynomials that share the properties of the classical polynomials of Jacobi, Hermite and Laguerre, as pointed out by Andrews and Askey [1]. Andrews and Askey [1] define a family of orthogonal polynomials to be classical if and only if it is a special case or a limiting case of the Askey-Wilson polynomials. The Askey-Wilson polynomials $\left\{p_{n}(x ; a, b, c, d \mid q\}\right.$ provide a basic generalization or a $q$-analog of Wigner's $6-j$ symbols and the Racah coefficients, [6, 29]. They satisfy the three-term recursion;

$$
\begin{gathered}
2 x p_{n}(x ; a, b, c, d \mid q)=A_{n} p_{n+1}(x ; a, b, c, d \mid q)+B_{n} p_{n}(x ; a, b, c, d \mid q) \\
+C_{n} p_{n-1}(x ; a, b, c, d \mid q), \\
A_{n}=\frac{a^{-1}\left(1-a b q^{n}\right)\left(1-a c q^{n}\right)\left(1-a d q^{n}\right)\left(1-a b c d q^{n-1}\right)}{\left(1-a b c d q^{2 n-1}\right)\left(1-a b c d q^{2 n}-q^{2 n}\right)}, \\
\quad C_{n}=\frac{a\left(1-b c q^{n-1}\right)\left(1-b d q^{n-1}\right)\left(1-c d q^{n-1}\right)\left(1-q^{n}\right)}{\left(1-a b c d q^{2 n-1}\right)\left(1-a b c d q^{2 n-2}\right)}, \\
B_{n}=a+a^{-1}-A_{n}-C_{n} .
\end{gathered}
$$

In general, if a sequence of orthogonal polynomials $\left\{p_{n}(x)\right\}$ satisfy an initial value problem:

$$
\begin{aligned}
& p_{0}(x)=1, \quad p_{1}(x)=\left(x-b_{0}\right) / a_{0}, \\
& x p_{n}(x)=a_{n} p_{n+1}(x)+b_{n} p_{n}(x)+c_{n} p_{n-1}(x), \quad n>0,
\end{aligned}
$$

then the associated polynomials $\left\{p_{n}^{\alpha}(x)\right\}$ are generated by

$$
\begin{aligned}
& p_{0}^{\alpha}(x)=1, \quad p_{1}^{\alpha}(x)=\left(x-b_{\alpha}\right) / a_{\alpha}, \\
& x p_{n}^{\alpha}(x)=a_{n+\alpha} p_{n+1}^{\alpha}(x)+b_{n+\alpha} p_{n}^{\alpha}(x)+c_{n+\alpha} p_{n-1}^{\alpha}(x), \quad n>0 .
\end{aligned}
$$

This is of interest when $\alpha$ is not necessarily a positive integer but the sequences $\left\{a_{n+\alpha}\right\},\left\{b_{n+\alpha}\right\},\left\{c_{n+1+\alpha}\right\}$ are well defined for $n=0,1, \ldots$, and for values of $\alpha$ in a certain connected subset of $(-\infty, \infty)$ containing $[0, \infty)$. This is particularly the case when $a_{n}$ 's, $b_{n}$ 's and $c_{n}$ 's are rational functions of $n$ or $q^{n}$. The associated polynomials are orthogonal if and only if the positivity condition

$$
a_{n+\alpha} c_{n+\alpha+1}>0, \quad n=0,1, \ldots,
$$

is satisfied, [ 5,7$]$. For recent work on associated polynomials we refer the reader to the articles $[5,8,10,14,15,19,25,33]$, and their references. 
In this paper we introduce two families of associated Askey-Wilson polynomials. Our approach is to use the properties of very well-poised basic hypergeometric series. The basic hypergeometric series in (1.3) is called well-poised if

$$
b_{1}=q a_{1} / a_{2}, \quad b_{2}=q a_{1} / a_{3}, \ldots, \quad b_{r}=q a_{1} / a_{r+1} ;
$$

it is called very well-poised if, in addition, $a_{2}=q a_{1}^{1 / 2}$ and $a_{3}=-q a_{1}^{1 / 2}$. Since the very well-poised ${ }_{8} \phi_{7}$ and ${ }_{10} \phi_{9}$ series will be used quite frequently in this paper, we shall use the contracted notation

$$
\begin{aligned}
& { }_{r+1} W_{r}\left(a ; a_{1}, \ldots, a_{r-2} ; q, z\right) \\
& \quad:={ }_{r+1} \phi_{r}\left[\begin{array}{c}
a, q a^{1 / 2},-q a^{1 / 2}, a_{1}, \ldots, a_{r-2} ; q, z \\
a^{1 / 2},-a^{1 / 2}, q a / a_{1}, \ldots, q a / a_{r-2}
\end{array}\right] .
\end{aligned}
$$

In $\S 2$ we shall first obtain a set of contiguous relations satisfied by

$$
{ }_{8} W_{7}\left(a ; b, c, d, e, f, ; q, a^{2} q^{2} / b c d e f\right)
$$

and use them in $\S 3$ to prove that two linearly independent solutions of the functional equation

$$
2 x h_{\alpha}(z)=A_{\alpha} h_{\alpha+1}(z)+B_{\alpha} h_{\alpha}(z)+C_{\alpha} h_{\alpha-1}(z)
$$

are given by

$$
\begin{aligned}
r_{\alpha}(z)= & \frac{\left(a b q^{\alpha}, a c q^{\alpha}, a d q^{\alpha}, b c d q^{\alpha} / z\right)_{\infty}}{\left(b c q^{\alpha}, b d q^{\alpha}, c d q^{\alpha}, a z q^{\alpha}\right)_{\infty}}\left(\frac{a}{z}\right)^{\alpha} \\
& \cdot{ }_{8} W_{7}\left(b c d / q z ; b / z, c / z, d / z, a b c d q^{\alpha-1}, q^{-\alpha} ; q, q z / a\right),
\end{aligned}
$$

and

$$
\begin{aligned}
s_{\alpha}(z)= & \frac{\left(a b c d q^{2 \alpha}, b z q^{\alpha+1}, c z q^{\alpha+1}, d z q^{\alpha+1}, b c d z q^{\alpha}\right)_{\infty}}{\left(b c q^{\alpha}, b d q^{\alpha}, c d q^{\alpha}, q^{\alpha+1}, b c d z q^{2 \alpha+1}\right)_{\infty}}(a z)^{\alpha} \\
& \cdot{ }_{8} W_{7}\left(b c d z q^{2 \alpha} ; b c q^{\alpha}, b d q^{\alpha}, c d q^{\alpha}, q^{\alpha+1}, z q / a ; q, a z\right),
\end{aligned}
$$

where

$$
\begin{gathered}
A_{\alpha}=a^{-1} \frac{\left(1-a b q^{\alpha}\right)\left(1-a c q^{\alpha}\right)\left(1-a d q^{\alpha}\right)\left(1-a b c d q^{\alpha-1}\right)}{\left(1-a b c d q^{2 \alpha-1}\right)\left(1-a b c d q^{2 \alpha}\right)} \\
C_{\alpha}=a \frac{\left(1-b c q^{\alpha-1}\right)\left(1-b d q^{\alpha-1}\right)\left(1-c d q^{\alpha-1}\right)\left(1-q^{\alpha}\right)}{\left(1-a b c d q^{2 \alpha-2}\right)\left(1-a b c d q^{2 \alpha-1}\right)}
\end{gathered}
$$

and

$$
B_{\alpha}=a+a^{-1}-A_{\alpha}-C_{\alpha},
$$

and $\alpha$ is an arbitrary complex parameter such that $\alpha \neq-1,-2, \ldots$, with $\left(z+z^{-1}\right) / 2=x$, as in (1.4). When $\alpha=n$, it can be shown by using the transformation formula of Watson [9, 8.5(2), p. 69] and Sears [26] that $r_{n}(x)$ is a multiple of the Askey-Wilson polynomials defined in (1.4). If $|z|<1$ and 
$|a|<1$ then the ${ }_{8} \phi_{7}$ series in (1.13) is convergent but the series in (1.12) may not converge. So, to ensure convergence of both these series in the nonterminating case we assume, for the time being, that $|z|<1$ and $|q|<|a|<1$. The assumption $|z|<1$ is equivalent to requiring $x$ to be in the complex plane cut along $[-1,1]$.

Masson [20] observed that when $a b c d=q$, or $q^{2}$ in the Askey-Wilson polynomials $(\alpha=0)$, there is an indeterminacy in the expressions (1.14), (1.15), (1.16) for $A_{\alpha}, C_{\alpha}, B_{\alpha}$, respectively. He pointed out that one can redefine $A_{0}, B_{0}, C_{0}$ as the limiting values of $A_{n}, B_{n}, C_{n}$ as $n \rightarrow 0^{+}$. This leads to a new family of polynomials that Masson called exceptional Askey-Wilson polynomials. Similarly a family of exceptional Jacobi polynomials was introduced and studied in [16]. Masson [20] pointed out that the continued $J$-fraction corresponding to the exceptional Wilson polynomials $(q=1)$ is in Ramanujan's published notebooks. Masson's exceptional Askey-Wilson polynomials correspond to the limiting case $\alpha \rightarrow 0^{+}$of $p_{n}^{\alpha}(x ; a, b, c, d \mid q)$ studied in this work.

A sequence of polynomials $\left\{p_{n}(x)\right\}$ generated by (1.7) is orthogonal with respect to a positive measure with infinite support and finite moments if and only if the positivity condition

$$
a_{n-1} c_{n}>0, \quad n=1,2, \ldots,
$$

holds. Furthermore, if $(1.17)$ holds and $\left\{p_{n}(x)\right\}$ is orthogonal with respect to a positive measure $d \mu$ then the orthogonality relation will be

$$
\int_{-\infty}^{\infty} p_{n}(x) p_{m}(x) d \mu(x)=\xi_{n} \delta_{m, n},
$$

where

$$
\xi_{0}=1, \quad \xi_{n}=\left(c_{1} c_{2} \cdots c_{n}\right) /\left(a_{0} a_{1} \cdots a_{n-1}\right), \quad n=1,2, \ldots
$$

In (1.18) the measure $d \mu$ has been normalized by

$$
\int_{-\infty}^{\infty} d \mu(t)=1
$$

To determine the weight function of the associated Askey-Wilson polynomials we found it convenient to use a theorem of Nevai [22, Corollary 36, p. 141, Theorem 40, p. 143] stated below.

Theorem 1. Assume that $\left\{p_{n}(x)\right\}$ is generated by (1.7) and that (1.17) and (1.18) hold. If the series $\sum_{n=1}^{\infty}\left\{\left|b_{n}\right|+\left|\sqrt{a_{n-1} b_{n}}-1 / 2\right|\right\}$ converges then $\left\{p_{n}(x)\right\}$ is orthogonal with respect to a positive measure

$$
d \mu(x)=\mu^{\prime}(x) d x+d j(x)
$$

where $j$ is a jump function, with possibly infinitely many jumps, and

$$
\limsup _{n} p_{n}^{2}(x) \mu^{\prime}(x) \sqrt{1-x^{2}} / \xi_{n}=\frac{2}{\pi},
$$


for almost all $x$ in $(-1,1)$, where $\xi_{n}$ is as in (1.19). Furthermore $j(x)$ is constant in $(-1,1)$.

Polynomials generated by (1.7) are denominators of approximants of positive definite $J$-fractions when $(1.17)$ is satisfied. The numerators of the corresponding approximants of the $J$-fractions, say $\left\{p_{n}^{*}(x)\right\}$, are generated by

$$
\begin{aligned}
p_{0}^{*}(x) & =0, \quad p_{1}^{*}(x)=1 / a_{0}, \\
x p_{n}^{*}(x) & =a_{n} p_{n+1}^{*}(x)+b_{n} p_{n}^{*}(x)+c_{n} p_{n-1}^{*}(x), \quad n>0 .
\end{aligned}
$$

If $\left\{a_{n}\right\},\left\{b_{n}\right\}$ and $\left\{c_{n}\right\}$ are bounded sequences then a theorem of Markov, (Szegö $[29, \S 3.5])$ asserts that:

$$
\lim _{n \rightarrow \infty} p_{n}^{*}(x) / p_{n}(x)=\int_{-\infty}^{\infty} \frac{d \mu(t)}{x-t}, \quad x \notin \operatorname{supp}\{d \mu\},
$$

uniformly on compact subsets of the complex plane cut along any closed interval containing the support of $d \mu$. Here $d \mu$ is normalized by (1.20). When we consider associated polynomials $\left\{p_{n}^{\alpha}(x)\right\}$, as in (1.8), then in view of (1.22) we have

$$
\left(p_{n}^{\alpha}(x)\right)^{*}=p_{n-1}^{\alpha+1}(x) / a_{\alpha}, \quad n>0 .
$$

If $\left\{a_{n+\alpha}\right\},\left\{b_{n+\alpha}\right\},\left\{c_{n+\alpha}\right\}$ are bounded, and $\left\{p_{n}^{\alpha}(x)\right\}$ is orthogonal with respect to $d \mu^{\alpha}$, then (1.23) and (1.24) yield

$$
\lim _{n \rightarrow \infty} p_{n-1}^{\alpha+1}(x) / p_{n}^{\alpha}(x)=a_{\alpha} \int_{-\infty}^{\infty} \frac{d \mu^{\alpha}(t)}{x-t}, \quad x \notin \operatorname{supp}\{d \mu\}
$$

The measure $d \mu^{\alpha}$ can then be found from (1.25) using the Perron-Stieltjes inversion formula for the Stieltjes transform.

A birth and death process with birth rates $\left\{\lambda_{n}\right\}$ and death rates $\left\{\mu_{n}\right\}$ generates a family of orthogonal polynomials $\left\{Q_{n}(x)\right\}$ via

$$
\begin{gathered}
Q_{0}(x)=1, \quad Q_{1}(x)=\left(\lambda_{0}+\mu_{0}-x\right) / \lambda_{0}, \\
-x Q_{n}(x)=\lambda_{n} Q_{n+1}(x)+\mu_{n} Q_{n-1}(x)-\left(\lambda_{n}+\mu_{n}\right) Q_{n}(x), \quad \\
\quad n=1,2, \ldots .
\end{gathered}
$$

It is assumed that

$$
\mu_{0} \geq 0, \lambda_{n}>0 \text { for } n \geq 0, \quad \mu_{n}>0 \text { for } n>0 .
$$

When $\mu_{n}$ is a given function $g(n)$, usually a polynomial or rational function, and $\mu_{0}=g(0) \neq 0$ there is usually a companion process with $\mu_{0}$ redefined as $\mu_{0}=0$. This companion process leads to a second family of orthogonal polynomials $\left\{q_{n}(x)\right\}$ generated by (1.27) and the initial conditions

$$
q_{0}(x)=1, \quad q_{1}(x)=\left(\lambda_{0}-x\right) / \lambda_{0} .
$$

When $\mu_{0}=0$ one can prove by induction that $Q_{n}(0)=1$, so the companion family $\left\{q_{n}(x)\right\}$ always satisfies

$$
q_{n}(0)=1, \quad n \geq 0 .
$$


The Askey-Wilson polynomials of (1.5) are renormalized birth and death polynomials for which $\mu_{0}$ vanishes. The associated Askey-Wilson polynomials arise from (1.11), (1.14), (1.15), (1.16) when $\alpha$ is replaced by $n+\alpha$. Since $\mu_{0}=C_{\alpha} \neq 0$, for $\alpha>0$ it makes good sense to introduce a second family of Askey-Wilson polynomials with the initial conditions (1.28). The second family of associated Askey-Wilson polynomials $\left\{q_{n}^{\alpha}(x)\right\}$ will be introduced in $\S 5$ where we express the $q_{n}^{\alpha}$ 's in terms of $r_{\alpha}(x)$ and $s_{\alpha}(x)$, the basis of solutions of the recurrence relation (1.11). An explicit formula for $q_{n}^{\alpha}(x)$ which exhibits its polynomial character will be derived in $\S 8$.

Note that when abcd is not equal to $q^{j}, j=1,2$, both families of associated Askey-Wilson polynomials tend to the Askey-Wilson polynomials as $\alpha \rightarrow 0^{+}$. It is interesting to note that if $a b c d=q$ or $a b c d=q^{2}$ then the family $\left\{q_{n}(x)\right\}$ tends to the Askey-Wilson polynomials while the $\left\{p_{n}^{\alpha}(x)\right\}$ tend to the exceptional families discussed by D. Masson [20].

T. Chihara [11] considered the effect of changing initial conditions on the spectral properties of the orthogonal polynomials generated by the same recurrence relation. Chihara considered general initial conditions. The change in initial conditions (1.28) from (1.26) may not be the most general but we believe it to be the most natural.

The presence of a second family of birth and death process polynomials was observed and investigated for linear and asymptotically symmetric quadratic processes in [14 and 15], respectively. Wimp [33] introduced and investigated associated Jacobi polynomials. The exceptional family of Jacobi polynomials was introduced by Ismail and Masson in [16].

In $\S 9$ we include miscellaneous results. The first is a complete asymptotic expansion of $\left\{p_{n}^{\alpha}(x)\right\}$. The second result, Theorem 4 , states that the coefficients $c_{m, n, k}^{\alpha}$ in the linearization of products

$$
\begin{aligned}
p_{m}^{\alpha}(x ; a, b, c, d \mid q) p_{n}^{\alpha}(x ; a, b, c, d \mid q) \\
\quad=\sum_{k=|m-n|}^{m+n} c_{m, n, k}^{\alpha}(a, b, c, d) p_{n}^{\alpha}(x ; a, b, c, d \mid q),
\end{aligned}
$$

are nonnegative for $\alpha \geq 0$, and $a=-b, c=-d,-q<b, d<q$. The linearization coefficients are usually very difficult to find explicitly, but in many cases of orthogonal polynomials the nonnegativity of the linearization coefficients has interesting implications (see the excellent source [3]). They may also have combinatorial interpretations (see [4]).

The following theorem of Askey [2] is very useful in proving the nonnegativity of linearization coefficients.

Theorem 2. Assume that $\left\{r_{n}(x)\right\}$ is a family of monic polynomials, i.e. $r_{n}(x)-x^{n}$ is a polynomial of degree less than $n$, and let $r_{0}(x)=1, r_{1}(x)=x+\alpha_{0}$, where $\alpha_{0}$ is a real number. If

$$
r_{1}(x) r_{n}(x)=r_{n+1}(x)+\alpha_{n} r_{n}(x)+\beta_{n} r_{n-1}(x), \quad n>0,
$$


and $\alpha_{n} \geq 0, \beta_{n+1}>0, \alpha_{n+1} \geq \alpha_{n}, \beta_{n+2} \geq \beta_{n+1}, n=0,1,2, \ldots$, then the coefficients $d_{m, n, k}$ in the linearization formula

$$
r_{m}(x) r_{n}(x)=\sum_{k=|m-n|}^{m+n} d_{m, n, k} r_{k}(x)
$$

are nonnegative.

There is very little known about the location of zeros of hypergeometric functions ${ }_{2} F_{1}$ or generalized hypergeometric functions ${ }_{p} F_{q}$ when the parameters are complex. There does not seem to be anything known about zeros of basic hypergeometric series (functions). Studying the presence, or absence, of a discrete spectrum for a family of orthogonal polynomials is a problem of determining when a certain transcendental function has, or does not have, zeros. In $\S 4$ we shall prove that $\left\{p_{n}^{\alpha}(x)\right\}$ is orthogonal with respect to an absolutely continuous measure when $\alpha \geq 0,-1<a, b, c, d<1,0<q<1$. In $\S 5$ we shall prove that $\left\{q_{n}^{\alpha}(x)\right\}$ is orthogonal with respect to a positive measure with at most one discrete mass which, if it exists, will lie outside $[-1,1]$. This proves the following theorem.

Theorem 3. Let $\alpha \geq 0,0<q<1$. If $-\sqrt{q}<a, b, c, d<\sqrt{q}$ then the ${ }_{8} W_{7}$ in (4.31) will have no zeros in $\mathbb{C} /[-1,1]$. On the other hand if $-1<a, b, c, d<$ 1 then the ${ }_{8} W_{7}$ in $(5.15)$ will have at most one zero in $\mathbb{C} /[-1,1]$.

\section{Contiguous Relations fOR ${ }_{8} W_{7}$ Series}

Let us assume that $a, d, e$ and $f$ are fixed parameters and denote

$$
\phi(b, c):={ }_{8} W_{7}\left(a ; b, c, d, e, f ; q, a^{2} q^{2} / b c d e f\right) .
$$

We shall use the usual notation of writing $b \pm$ for $b q^{ \pm 1}, \ldots$, etc. For example $\phi(b \pm, c)$ stands for $\phi\left(b q^{ \pm 1}, c\right)$ and $\phi(b, c \pm)$ for $\phi\left(b, c q^{ \pm 1}\right)$. A contiguous relation is a three term recurrence relation connecting $\phi(b, c)$, $\phi(b \pm, c \pm)$. We shall first prove the following identities:

$$
\begin{aligned}
(b-c) & \left(1-a^{2} q^{2} / b c d e f\right) \phi(b, c) \\
= & b \frac{(1-a q / b d)(1-a q / b e)(1-a q / b f)}{(1-a q / b)} \phi(b-, c) \\
& -c \frac{(1-a q / c d)(1-a q / c e)(1-a q / c f)}{(1-a q / c)} \phi(b, c-),
\end{aligned}
$$

and

$$
\begin{aligned}
(c-b / q)(1-a q / b c) \phi(b-, c)= & (c-1)(1-a / c) \phi(b-, c+) \\
& +(1-b / q)(1-a q / b) \phi(b, c) .
\end{aligned}
$$


To prove (2.2) we shall use Nassrallah and Rahman's [21] integral representation for an ${ }_{8} W_{7}$ series:

$$
\begin{aligned}
\int_{-1}^{1} v & (x ; \lambda, \mu, \nu, \rho, \sigma ; A) d x \\
= & \frac{2 \pi\left(A \lambda, A \mu, A \nu, A \rho, A \sigma, \lambda \mu \nu \rho \sigma A^{-1}\right)_{\infty}}{\left(q, \lambda \mu, \lambda \nu, \lambda \rho, \lambda \sigma, \mu \nu, \mu \rho, \mu \sigma, \nu \rho, \nu \sigma, \rho \sigma, A^{2}\right)_{\infty}} \\
& \cdot{ }_{8} W_{7}\left(A^{2} / q ; A / \lambda, A / \mu, A / \nu, A / \rho, A / \sigma ; q, \lambda \mu \nu \rho \sigma A^{-1}\right),
\end{aligned}
$$

provided that

$$
\operatorname{Max}\left(|\lambda|,|\mu|,|\nu|,|\rho|,|\sigma|,\left|\lambda \mu \nu \rho \sigma A^{-1}\right|\right)<1
$$

where

$$
\begin{aligned}
& v(x ; \lambda, \mu, \nu, \rho, \sigma ; A) \\
& \quad=\frac{h\left(x ; 1,-1, q^{1 / 2},-q^{1 / 2}, A\right)}{h(x ; \lambda, \mu, \nu, \rho, \sigma)}\left(1-x^{2}\right)^{-1 / 2},
\end{aligned}
$$

with

$$
\begin{aligned}
& h\left(x ; a_{1}, a_{2}, \ldots, a_{k}\right)=\prod_{j=1}^{k} h\left(x ; a_{j}\right), \\
& h(x ; a)=\prod_{n=0}^{\infty}\left(1-2 a x q^{n}+a^{2} q^{2 n}\right) .
\end{aligned}
$$

Since $1-2 A x+A^{2}=(1-A / \lambda)(1-A \lambda)+A\left(1-2 \lambda x+\lambda^{2}\right) / \lambda$ and $h(x ; A)=$ $\left(1-2 A x+A^{2}\right) h(x ; q A)$, the integral in (2.4) can be split into two parts:

$$
\begin{aligned}
\int_{-1}^{1} v & (x ; \lambda, \mu, \nu, \rho, \sigma ; A) d x \\
= & (1-A / \lambda)(1-A \lambda) \int_{-1}^{1} v(x ; \lambda, \mu, \nu, \rho, \sigma ; A q) d x \\
& +\frac{A}{\lambda} \int_{-1}^{1} v(x ; \lambda q, \mu, \nu, \rho, \sigma ; A q) d x .
\end{aligned}
$$

This decomposition leads to the following identity involving ${ }_{8} W_{7}$ series:

$$
\begin{aligned}
&{ }_{8} W_{7}\left(A^{2} / q ; A / \lambda, A / \mu, A / \nu, A / \rho, A / \sigma ; q, \lambda \mu \nu \rho \sigma / A\right) \\
&=(1-A / \lambda) \frac{\left(1-A^{2}\right)\left(1-q A^{2}\right)(1-\lambda \mu \nu \rho \sigma / q A)}{(1-A \mu)(1-A \nu)(1-A \rho)(1-A \sigma)} \\
& \cdot{ }_{8} W_{7}\left(q A^{2} ; A q / \lambda, A q / \mu, A q / \nu, A q / \rho, A q / \sigma ; q, \frac{\lambda \mu \nu \rho \sigma}{A q}\right) \\
&+\frac{A}{\lambda} \frac{\left(1-A^{2}\right)\left(1-q A^{2}\right)(1-\lambda \mu)(1-\lambda \nu)(1-\lambda \rho)(1-\lambda \sigma)}{(1-A \lambda)(1-A \lambda q)(1-A \mu)(1-A \nu)(1-A \rho)(1-A \sigma)} \\
& \cdot{ }_{8} W_{7}\left(q A^{2} ; A / \lambda, q A / \mu, q A / \nu, q A / \rho, q A / \sigma ; q, \frac{\lambda \mu \nu \rho \sigma}{A}\right) .
\end{aligned}
$$


We then interchange $\lambda$ and $\mu$ in (2.8) and subtract the result from (2.8). After replacing $A$ by $A / q$ we obtain the functional equation

$$
\begin{aligned}
&(\lambda-\mu)(1-\lambda \mu \nu \rho \sigma / A)_{8} W_{7}\left(A^{2} / q ; A / \lambda, A / \mu, A / \nu, A / \rho, A / \sigma ; q, \lambda \mu \nu \rho \sigma / A\right) \\
&= \frac{\lambda(1-\mu \nu)(1-\mu \rho)(1-\mu \sigma)}{1-A \mu} \\
& \cdot{ }_{8} W_{7}\left(A^{2} / q ; A / \lambda, A / \mu q, A / \nu, A / \rho, A / \sigma ; q, \lambda \mu \nu \rho \sigma q / A\right) \\
&-\frac{\mu(1-\lambda \nu)(1-\lambda \rho)(1-\mu \sigma)}{1-A \lambda} \\
& \cdot{ }_{8} W_{7}\left(A^{2} / q ; A / \lambda q, A / \mu, A / \nu, A / \rho, A / \sigma ; q, \lambda \mu \nu \rho \sigma q / A\right) .
\end{aligned}
$$

Identity (2.2) follows from (2.9) by setting $A=(a q)^{1 / 2}, \lambda=(a q)^{1 / 2} / b$, $\mu=(a q)^{1 / 2} / c, \nu=(a q)^{1 / 2} / d, \rho=(a q)^{1 / 2} / e$, and $\sigma=(a q)^{1 / 2} / f$.

To prove (2.3) we observe that for $n=0,1,2, \ldots$, we have

$$
\begin{aligned}
\frac{\left(a q^{n+2} / b, a q^{n} / c\right)_{\infty}}{\left(b q^{n-1}, c q^{n+1}\right)_{\infty}}-\frac{\left(a q^{n+1} / b, a q^{n+1} / c\right)_{\infty}}{\left(b q^{n}, c q^{n}\right)_{\infty}} \\
=\frac{\left(a q^{n+2} / b, a q^{n+1} / c\right)_{\infty}}{\left(b q^{n-1}, c q^{n}\right)_{\infty}} \\
\cdot\left\{\left(1-a q^{n} / c\right)\left(1-c q^{n}\right)-\left(1-a q^{n+1} / b\right)\left(1-b q^{n-1}\right)\right\} \\
=(b / q-c)(1-a q / b c) \frac{\left(a q^{n+2} / b, a q^{n+1} / c\right)_{\infty}}{\left(b q^{n-1}, c q^{n}\right)_{\infty}} q^{n} .
\end{aligned}
$$

This establishes the contiguous relation

$$
\begin{gathered}
\frac{\left(a q^{2} / b, a / c\right)_{\infty}}{(b / q, c q)_{\infty}} \phi(b-, c+)-\frac{(a q / b, a q / c)_{\infty}}{(b, c)_{\infty}} \phi(b, c) \\
\quad=(b / q-c)(1-a q / b c) \frac{\left(a q^{2} / b, a q / c\right)_{\infty}}{(b / q, c)_{\infty}} \phi(b-, c),
\end{gathered}
$$

which is essentially the same as (2.3).

Let us now rewrite $(2.3)$ in the form

$$
\begin{aligned}
\phi(b-, c)= & \frac{(1-c)(1-a / c)}{(b / q-c)(1-a q / b c)} \phi(b-, c+) \\
& -\frac{(1-b / q)(1-a q / b)}{(b / q-c)(1-a q / b c)} \phi(b, c) .
\end{aligned}
$$

Interchanging $b$ and $c$ we also have

$$
\begin{aligned}
\phi(b, c-)= & \frac{(1-b)(1-a / b)}{(c / q-b)(1-a q / b c)} \phi(b+, c-) \\
& -\frac{(1-c / q)(1-a q / c)}{(c / q-b)(1-a q / b c)} \phi(b, c) .
\end{aligned}
$$


We now multiply $(2.11)$ by $b(1-a q / b d)(1-a q / b e)(1-a q / b f) /(1-a q / b)$, multiply $(2.12)$ by $c(1-a q / c d)(1-a q / c e)(1-a q / c f) /(1-a q / c)$, then subtract one from the other and use (2.2) to get the contiguous relation

$$
\begin{aligned}
& {\left[(b-c)(1-a q / b c)\left(1-a^{2} q^{2} / b c d e f\right)+L+M\right] \phi(b, c)} \\
& \quad=\frac{(1-c)(1-a / c) M}{(1-b / q)(1-a q / b)} \phi(b-, c+)+\frac{(1-b)(1-a / b) L}{(1-c / q)(1-a q / c)} \phi(b+, c-),
\end{aligned}
$$

where

$$
L=\frac{c(1-c / q)(1-a q / c d)(1-a q / c e)(1-a q / c f)}{b-c / q}
$$

and

$$
M=\frac{b(1-b / q)(1-a q / b d)(1-a q / b e)(1-a q / b f)}{b / q-c} .
$$

As we shall see in the next section, (2.13) enables us to show that $r_{\alpha}(x)$ defined in (1.12) is a solution of the functional equation (1.11). However, in order to show that $s_{\alpha}(x)$ defined in (1.13) also satisfies (1.11) we need a slightly different approach. Let us assume that $\mu, \nu, \rho, \sigma$ are fixed constants and set

$$
W(A ; \lambda):={ }_{8} W_{7}\left(A^{2} / q ; A / \lambda, A / \mu, A / \nu, A / \rho, A / \sigma ; q, \lambda \mu \nu \rho \sigma / A\right) .
$$

We then replace $A, \lambda$ in $(2.8)$ by $A / q$ and $\lambda / q$, respectively, and write it in the form

$$
\begin{aligned}
& W(A / q ; \lambda / q)=\frac{(1-A / \lambda)\left(1-A^{2} / q^{2}\right)\left(1-A^{2} / q\right)(1-\lambda \mu \nu \rho \sigma / q A)}{(1-A \mu / q)(1-A \nu / q)(1-A \rho / q)(1-A \sigma / q)} W(A ; \lambda / q) \\
& \quad+\frac{A}{\lambda} \frac{\left(1-A^{2} / q\right)\left(1-A^{2} / q^{2}\right)(1-\lambda \mu / q)(1-\lambda \nu / q)(1-\lambda \rho / q)(1-\lambda \sigma / q)}{\left(1-A \lambda / q^{2}\right)(1-A \lambda / q)(1-A \mu / q)(1-A \nu / q)(1-A \rho / q)(1-A \sigma / q)} W(A ; \lambda) .
\end{aligned}
$$

By using the definition of the ${ }_{8} \phi_{7}$ series it can be easily verified that (2.18)

$$
\begin{aligned}
& W(A ; \lambda / q)-W(A ; \lambda) \\
& =\frac{\left(1-A^{2}\right)\left(1-q A^{2}\right)(1-A / \mu)(1-A / \nu)(1-A / \rho)(1-A / \sigma)}{(1-A \lambda / q)(1-A \lambda)(1-A \mu)(1-A \nu)(1-A \rho)(1-A \sigma)} \frac{\lambda \mu \nu \rho \sigma}{A q} \\
& \quad \cdot W(A q ; \lambda) .
\end{aligned}
$$

Eiminating $W(A ; \lambda / q)$ between $(2.17)$ and (2.18) we find that

$$
\begin{aligned}
& W(A / q ; \lambda / q) \\
& =\left[\frac{(1-A / \lambda)\left(1-A^{2} / q^{2}\right)\left(1-A^{2} / q\right)(1-\lambda \mu \nu \rho \sigma / A q)}{(1-A \mu / q)(1-A \nu / q)(1-A \rho / q)(1-A \sigma / q)}\right. \\
& \left.+\frac{A}{\lambda} \frac{(1-\lambda \mu / q)(1-\lambda \nu / q)(1-\lambda \rho / q)(1-\lambda \sigma / q)\left(A^{2} / q^{2}\right)_{2}}{(1-A \mu / q)(1-A \nu / q)(1-A \rho / q)(1-A \sigma / q)\left(A \lambda / q^{2}\right)_{2}}\right] W(A ; \lambda) \\
& \quad+\frac{\lambda \mu \nu \rho \sigma}{A q} \frac{(1-A / \lambda)(1-A / \mu)(1-A / \nu)(1-A / \rho)(1-A / \sigma)(1-\lambda \mu \nu \rho \sigma / A q)\left(A^{2} / q^{2}\right)_{4}}{\left(A \lambda / q_{2}\right)(A \mu / q)_{2}(A \nu / q)_{2}(A \rho / q)_{2}(A \sigma / q)_{2}} \\
& \quad \cdot W(A q ; \lambda) .
\end{aligned}
$$


We can now express $W(A q ; \lambda)$ in terms of $W(A ; \lambda)$ and $W(A q ; \lambda q)$ by using (2.17) with $A, \lambda$ replaced by $A q, \lambda q$, respectively. We thus obtain the second independent contiguous relation for the ${ }_{8} W_{7}$ series

$$
\begin{aligned}
{\left[(1-A / \lambda)(1-\lambda \mu \nu \rho \sigma / A q)+\frac{A}{\lambda} \frac{(1-\lambda \mu / q)(1-\lambda \nu / q)(1-\lambda \rho / q)(1-\lambda \sigma / q)}{\left(1-A \lambda / q^{2}\right)(1-A \lambda / q)}\right.} \\
\left.\quad+\frac{\lambda \mu \nu \rho \sigma}{A q} \frac{(1-A / \mu)(1-A / \nu)(1-A / \rho)(1-A / \sigma)}{(1-A \lambda / q)(1-A \lambda)}\right] W(A ; \lambda) \\
=\frac{(1-A \mu / q)(1-A \nu / q)(1-A \rho / q)(1-A \sigma / q)}{\left(1-A^{2} / q^{2}\right)\left(1-A^{2} / q\right)} W(A / q ; \lambda / q) \\
\quad+\frac{\mu \nu \rho \sigma}{q} \frac{\left(A^{2}\right)_{2}(1-A / \mu)(1-A / \nu)(1-A / \rho)(1-A / \sigma)(1-\lambda \nu)(1-\lambda \rho)(1-\lambda \sigma)}{(A \lambda / q)_{2}(A \lambda)_{2}(1-A \mu)(1-A \nu)(1-A \rho)(1-A \sigma)} \\
\quad+(1-\lambda \mu) W(A q ; \lambda q) .
\end{aligned}
$$

\section{Solutions OF THE FUNCTIONAL EQUATION (1.11)}

In (2.13) let us replace $a, b, c, d, e, f$ by $b c d / z q, q^{-\alpha}, a b c d q^{\alpha-1}, b / z$, $c / z$ and $d / z$, respectively, and set

$$
f_{\alpha}^{\prime}(z)={ }_{8} W_{7}\left(b c d / z q ; b / z, c / z, d / z, a b c d q^{\alpha-1}, q^{-\alpha} ; q, q z / a\right) .
$$

Then $(2.13)$ can be written as

$$
\begin{aligned}
(z+ & \left.z^{-1}-a / q-q / a+A_{\alpha}^{\prime}+C_{\alpha}^{\prime}\right) f_{\alpha}^{\prime}(z) \\
= & \frac{q}{a z} \frac{\left(1-a z q^{\alpha}\right)\left(1-a b c d q^{\alpha-1}\right)}{\left(1-b c d q^{\alpha} / z\right)\left(1-q^{\alpha+1}\right)} A_{\alpha}^{\prime} f_{\alpha+1}^{\prime}(z) \\
& +\frac{a z}{q} \frac{\left(1-b c d q^{\alpha-1} / z\right)\left(1-q^{\alpha}\right)}{\left(1-a z q^{\alpha-1}\right)\left(1-a b c d q^{\alpha-2}\right)} C_{\alpha}^{\prime} f_{\alpha-1}^{\prime}(z),
\end{aligned}
$$

where

$$
\begin{aligned}
& A_{\alpha}^{\prime}=\frac{a}{q} \frac{\left(1-b c q^{\alpha}\right)\left(1-b d q^{\alpha}\right)\left(1-c d q^{\alpha}\right)\left(1-q^{\alpha+1}\right)}{\left(1-a b c d q^{2 \alpha-1}\right)\left(1-a b c d q^{2 \alpha}\right)}, \\
& C_{\alpha}^{\prime}=\frac{q}{a} \frac{\left(1-a b q^{\alpha-1}\right)\left(1-a c q^{\alpha-1}\right)\left(1-a d q^{\alpha-1}\right)\left(1-a b c d q^{\alpha-2}\right)}{\left(1-a b c d q^{2 \alpha-2}\right)\left(1-a b c d q^{2 \alpha-1}\right)} .
\end{aligned}
$$

Similarly, replacing $A, \lambda, \mu, \nu, \rho, \sigma$ in $(2.20)$ by $(b c d z q)^{1 / 2} q^{\alpha}$, $a q^{\alpha}(b c d / z q)^{1 / 2},(d z q / b c)^{1 / 2},(c z q / b d)^{1 / 2},(b z q / c d)^{1 / 2}$, and $(b c d z / q)^{1 / 2}$ respectively, we find that $(2.20)$ can be rewritten as

$$
\begin{aligned}
\left(z+z^{-1}-a / q-q / a+A_{\alpha}^{\prime}+C_{\alpha}^{\prime}\right) g_{\alpha}^{\prime}(z) & \\
= & z A_{\alpha}^{\prime} C_{\alpha+1}^{\prime} \frac{\left(1-b c d z q^{2 \alpha+1}\right)\left(1-b c d z q^{2 \alpha+2}\right)}{\left(1-b z q^{\alpha+1}\right)\left(1-c z q^{\alpha+1}\right)\left(1-d z q^{\alpha+1}\right)\left(1-b c d z q^{\alpha}\right)} g_{\alpha+1}^{\prime}(z) \\
& +z^{-1} \frac{\left(1-b z q^{\alpha}\right)\left(1-c z q^{\alpha}\right)\left(1-d z q^{\alpha}\right)\left(1-b c d z q^{\alpha-1}\right)}{\left(1-b c d z q^{2 \alpha-1}\right)\left(1-b c d z q^{2 \alpha}\right)} g_{\alpha-1}^{\prime}(z),
\end{aligned}
$$


where

$$
g_{\alpha}^{\prime}(z)={ }_{8} W_{7}\left(b c d z q^{2 \alpha} ; b c q^{\alpha}, b d q^{\alpha}, c d q^{\alpha}, q^{\alpha+1}, z q / a ; q, a z\right) .
$$

If we set

$$
f_{\alpha}(z)=\frac{\left(q^{\alpha+1}, b c d q^{\alpha} / z\right)_{\infty}}{\left(a b c d q^{\alpha-1}, a z q^{\alpha}\right)_{\infty}}\left(\frac{q}{a z}\right)^{\alpha} f_{\alpha}^{\prime}(z)
$$

and

$$
g_{\alpha}(z)=\frac{\left(a b c d q^{2 \alpha}, b z q^{\alpha+1}, c z q^{\alpha+1}, d z q^{\alpha+1}, b c d z q^{\alpha}\right)_{\infty}}{\left(a b q^{\alpha}, a c q^{\alpha}, a d q^{\alpha}, a b c d q^{\alpha-1}, b c d z q^{2 \alpha+1}\right)_{\infty}}\left(\frac{q z}{a}\right)^{\alpha} g_{\alpha}^{\prime}(z)
$$

then it can be shown that both $f_{\alpha}(z)$ and $g_{\alpha}(z)$ satisfy the same functional equation, namely,

$$
\left(z+z^{-1}-a / q-q / a+A_{\alpha}^{\prime}+C_{\alpha}^{\prime}\right) h_{\alpha}^{\prime}(z)=A_{\alpha}^{\prime} h_{\alpha+1}^{\prime}(z)+C_{\alpha}^{\prime} h_{\alpha-1}^{\prime}(z) .
$$

To deduce the functional equation (1.11) we observe that

$$
A_{\alpha}^{\prime}+C_{\alpha}^{\prime}-a / q-q / a=A_{\alpha}+C_{\alpha}-a-a^{-1}
$$

where

$$
A_{\alpha}=a^{-1} \frac{\left(1-a b q^{\alpha}\right)\left(1-a c q^{\alpha}\right)\left(1-a d q^{\alpha}\right)\left(1-a b c d q^{\alpha-1}\right)}{\left(1-a b c d q^{2 \alpha-1}\right)\left(1-a b c d q^{2 \alpha}\right)}
$$

and

$$
C_{\alpha}=a \frac{\left(1-b c q^{\alpha-1}\right)\left(1-b d q^{\alpha-1}\right)\left(1-c d q^{\alpha-1}\right)\left(1-q^{\alpha}\right)}{\left(1-a b c d q^{2 \alpha-2}\right)\left(1-a b c d q^{2 \alpha-1}\right)} .
$$

If we now define

$$
h_{\alpha}(z)=\frac{\left(a b q^{\alpha}, a c q^{\alpha}, a d q^{\alpha}, a b c d q^{\alpha-1}\right)_{\infty}}{\left(b c q^{\alpha}, b d q^{\alpha}, c d q^{\alpha}, q^{\alpha+1}\right)_{\infty}}\left(\frac{a^{2}}{q}\right)^{\alpha} h_{\alpha}^{\prime}(z),
$$

then it follows that (3.8) reduces to (1.11). Multiplying $f_{\alpha}(z)$ and $g_{\alpha}(z)$ by the coefficient of $h_{\alpha}^{\prime}(z)$ in (3.12) we find that $r_{\alpha}(z)$ and $s_{\alpha}(z)$ defined in (1.12) and (1.13), respectively, are indeed solutions of (1.11).

\section{The associated AsKey-Wilson polynomials}

Let $n$ be a nonnegative integer and let

$$
p_{n}^{\alpha}(x)=p_{n}^{\alpha}(x ; a, b, c, d \mid q)=L_{\alpha} r_{\alpha+n}(z)+M_{\alpha} s_{\alpha+n}(z),
$$

be a linear combination of solutions of (1.11) with $\alpha$ replaced by $\alpha+n$. The initial conditions to be used are

$$
p_{-1}^{\alpha}(x)=0, \quad p_{0}^{\alpha}(x)=1 .
$$

These initial conditions force the linear combination in (4.1) to depend on $z$ only through the combination $\left(z+z^{-1}\right) / 2=x$ and hence to be a polynomial in $x$. 
From the functional equation (1.11) it clearly follows that

$$
L_{\alpha}=W_{\alpha}^{-1} s_{\alpha-1}(z), \quad M_{\alpha}=-W_{\alpha}^{-1} r_{\alpha-1},
$$

where the Wronskian-type function $W_{\alpha}$ is given by the Casorati determinant

$$
W_{\alpha}=r_{\alpha}(z) s_{\alpha-1}(z)-s_{\alpha}(z) r_{\alpha-1}(z) \text {. }
$$

The nonvanishing of $W_{\alpha}$ provides the guarantee that $r_{\alpha}(z)$ and $s_{\alpha}(z)$ are linearly independent. To compute $W_{\alpha}$ we find from (1.11) that

$$
\begin{aligned}
& W_{\alpha}=A_{\alpha} W_{\alpha+1} / C_{\alpha} \\
& =a^{-2} \frac{\left(1-a b q^{\alpha}\right)\left(1-a c q^{\alpha}\right)\left(1-a d q^{\alpha}\right)\left(1-a b c d q^{\alpha-1}\right)\left(1-a b c d q^{2 \alpha-2}\right)}{\left(1-b c q^{\alpha-1}\right)\left(1-b d q^{\alpha-1}\right)\left(1-c d q^{\alpha-1}\right)\left(1-q^{\alpha}\right)\left(1-a b c d q^{2 \alpha}\right)} W_{\alpha+1} .
\end{aligned}
$$

Hence, for $V_{\alpha}=a^{-2 \alpha} W_{\alpha}$, we obtain

$$
V_{\alpha}=\frac{\left(1-a b q^{\alpha}\right)\left(1-a c q^{\alpha}\right)\left(1-a d q^{\alpha}\right)\left(1-a b c d q^{\alpha-1}\right)\left(1-a b c d q^{2 \alpha-2}\right)}{\left(1-b c q^{\alpha-1}\right)\left(1-b d q^{\alpha-1}\right)\left(1-c d q^{\alpha-1}\right)\left(1-q^{\alpha}\right)\left(1-a b c d q^{2 \alpha}\right)} V_{\alpha+1},
$$

which, on iteration, gives

$$
V_{\alpha}=\frac{\left(a b q^{\alpha}, a c q^{\alpha}, a d q^{\alpha}, a b c d q^{\alpha-1}\right)_{\infty}}{\left(b c q^{\alpha-1}, b d q^{\alpha-1}, c d q^{\alpha-1}, q^{\alpha}\right)_{\infty}}\left(1-a b c d q^{2 \alpha-2}\right) \lim _{n \rightarrow \infty} V_{\alpha+n}
$$

From (1.12), (1.13) and (4.4) it follows that

$$
\begin{aligned}
& \lim _{n \rightarrow \infty} V_{\alpha+n}= \lim _{n \rightarrow \infty} a^{-2(n+\alpha)}\left\{r_{\alpha+n}(z) s_{\alpha+n-1}(z)-r_{\alpha+n-1}(z) s_{\alpha+n}(z)\right\} \\
&=a^{-1} \lim _{n \rightarrow \infty}\left\{z^{-1}{ }_{8} W_{7}\left(b c d / z q ; b / z, c / z, d / z, a b c d q^{n+\alpha-1}, q^{-n-\alpha} ; q, z q / a\right)\right. \\
&{ }_{8} W_{7}\left(b c d z q^{2 n+2 d-2} ; b c q^{\alpha+n-1}, b d q^{\alpha+n-1}, c d q^{\alpha+n-1}, q^{\alpha+n-1}, z q / a ; q, a z\right) \\
& \quad-z_{8} W_{7}\left(b c d / z q ; b / z, c / z, d / z, a b c d q^{n+\alpha-2}, q^{1-n-\alpha} ; q, z q / a\right) \\
&\left.\quad{ }_{8} W_{7}\left(b c d z q^{2 n+2 \alpha} ; b c q^{\alpha+n}, b d q^{\alpha+n}, c d q^{\alpha+n}, q^{\alpha+n}, z q / a ; q, a z\right)\right\} .
\end{aligned}
$$

Now, for fixed $\beta$, the limiting relation

$$
\begin{aligned}
& \lim _{n \rightarrow \infty}{ }_{8} W_{7}\left(b c d z q^{2 n+2 \beta} ; b c q^{n+\beta}, b d q^{n+\beta}, c d q^{n+\beta}, q^{n+\beta}, z q / a ; q, a z\right) \\
& \quad=\frac{\left(q z^{2}\right)_{\infty}}{(a z)_{\infty}}, \quad|a z|<1,
\end{aligned}
$$

follows from the $q$-binomial theorem [27, IV.11], or [13, II.3, p. 236], while the limiting relation

$$
\begin{aligned}
& \lim _{n \rightarrow \infty}{ }_{8} W_{7}\left(b c d / z q ; b / z, c / z, d / z, a b c d q^{n+\beta-1}, q^{-n-\beta} ; q, z q / a\right) \\
& \quad={ }_{6} \phi_{5}\left[\begin{array}{r}
\left.b c d / z q, q(b c d / z q)^{1 / 2},-q(b c d / z q)^{1 / 2}, b / z, c / z, d / z ; q, z^{2}\right] \\
(b c d / z q)^{1 / 2},-(b c / z q)^{1 / 2}, c d, b d, b c
\end{array}\right. \\
&= \frac{(b c d / z, b z, c z, d z)_{\infty}}{\left(c d, b d, b c, z^{2}\right)_{\infty}}, \quad|z|<1,
\end{aligned}
$$


follows from the ${ }_{6} \phi_{5}$ summation formula [27, IV.7] or [13, II.20]. This yields the limiting relation

$$
\lim _{n \rightarrow \infty} V_{\alpha+n}=\frac{(b z, c z, d z, b c d / z)_{\infty}}{(b c, b d, c d, a z)_{\infty}}(a z)^{-1}
$$

Thus,

$$
\begin{aligned}
W_{\alpha}= & a^{2 \alpha} V_{\alpha} \\
= & \frac{a^{2 \alpha-1}}{z} \frac{\left(b z, c z, d z, b c d / z, a b q^{\alpha}, a c q^{\alpha}, a d q^{\alpha}, a b c d q^{\alpha-1}\right)_{\infty}}{\left(a z, b c, b d, c d, b c q^{\alpha-1}, c d q^{\alpha-1}, b d q^{\alpha-1}, q^{\alpha}\right)_{\infty}} \\
& \cdot\left(1-a b c d q^{2 \alpha-2}\right) .
\end{aligned}
$$

The associated Askey-Wilson polynomials are then given by the explicit formula (4.13)

$$
\begin{aligned}
& P_{n}^{\alpha}(x)=\frac{z a^{n}}{\left(1-a b c d q^{2 \alpha-2}\right)} \\
& \cdot \frac{\left(b c, b d, c d, b c q^{\alpha-1}, b d q^{\alpha-1}, c d q^{\alpha-1}, q^{\alpha}, a z\right)_{\infty}}{\left(a b q^{\alpha}, a c q^{\alpha}, a d q^{\alpha}, a b c d q^{\alpha-1}, b z, c z, d z, b c d / z\right)_{\infty}} \\
& \cdot\left\{\frac{\left(a b c d q^{2 \alpha-2}, b z q^{\alpha}, c z q^{\alpha}, d z q^{\alpha}, b c d z q^{\alpha-1}, a b q^{\alpha+n}\right)_{\infty}}{\left(b c q^{\alpha-1}, b d q^{\alpha-1}, c d q^{\alpha-1}, q^{\alpha}, b c d z q^{2 \alpha-1}, b c q^{\alpha+n}\right)_{\infty}}\right. \\
& \cdot \frac{\left(a c q^{\alpha+n}, a d q^{\alpha+n}, b c d q^{\alpha+n} / z\right)_{\infty}}{\left(b d q^{\alpha+n}, c d q^{\alpha+n}, a z q^{\alpha+n}\right)_{\infty}} z^{-n-1} \\
& \cdot{ }_{8} W_{7}\left(b c d z q^{2 \alpha-2} ; b c q^{\alpha-1}, b d q^{\alpha-1}, c d q^{\alpha-1}, q^{\alpha}, z q / a ; q, a z\right) \\
& { }_{8} W_{7}\left(b c d / z q ; b / z, c / z, d / z, a b c d q^{\alpha+n-1}, q^{-\alpha-n} ; q, q z / a\right) \\
& -\frac{\left(a b c d q^{2 \alpha+2 n}, b z q^{\alpha+n+1}, c z q^{\alpha+n+1}\right)_{\infty}}{\left(b c q^{\alpha+n}, b d q^{\alpha+n}, c d q^{\alpha+n}, q^{\alpha+n+1}\right)_{\infty}} \\
& \cdot \frac{\left(d z q^{\alpha+n+1}, b c d z q^{\alpha+n}, a b q^{\alpha-1}, a c q^{\alpha-1}, a d q^{\alpha-1}, b c d q^{\alpha-1} / z\right)_{\infty}}{\left(b c d z q^{2 \alpha+2 n+1}, b c q^{\alpha-1}, b d q^{\alpha-1}, c d q^{\alpha-1}, a z q^{\alpha-1}\right)_{\infty}} \\
& \cdot z^{n+1}{ }_{8} W_{7}\left(b c d z q^{2 \alpha+2 n} ; b c q^{\alpha+n}, b d q^{\alpha+n}, c d q^{\alpha+n}, q^{\alpha+n+1}, z q / a ; q, a z\right) \\
& \left.\cdot{ }_{8} W_{7}\left(b c d / z q ; b / z, c / z, d / z, a b c d q^{\alpha-2}, q^{1-\alpha} ; q, q z / a\right)\right\} .
\end{aligned}
$$

Using (4.1), (4.3) and (4.4) in (1.11) one can also show that $p_{n}^{\alpha}(x)$ satisfies the three-term recurrence relation

(4.14) $\left(z+z^{-1}-a-a^{-1}+A_{\alpha+n}+C_{\alpha+n}\right) p_{n}^{\alpha}(x)=A_{\alpha+n} p_{n+1}^{\alpha}(x)+C_{\alpha+n} p_{n-1}^{\alpha}(x)$.

Although (4.13) is the more useful form of $p_{n}^{\alpha}(x)$ it is far from clear that it is a polynomial in $\left(z+z^{-1}\right) / 2$. It is possible, in principle, to use the transformation theory of basic hypergeometric series to derive an explicit polynomial form of 
$p_{n}^{\alpha}(x)$ from (4.13), but we shall use a simpler approach in $\S 6$ to show that

$$
\begin{aligned}
& p_{n}^{\alpha}(x)= \sum_{k=0}^{n} \frac{\left(q^{-n}, a b c d q^{2 \alpha+n-1}, a b c d q^{2 \alpha-1}, a z, a / z\right)_{k}}{\left(q, a b q^{\alpha}, a c q^{\alpha}, a d q^{\alpha}, a b c d q^{\alpha-1}\right)_{k}} q^{k} \\
& \cdot{ }_{10} W_{9}\left(a b c d q^{2 \alpha+k-2} ; q^{\alpha}, b c q^{\alpha-1}, b d q^{\alpha-1}, c d q^{\alpha-1},\right. \\
&\left.q^{k+1}, a b c d q^{2 \alpha+n+k-1}, q^{k-n} ; q, a^{2}\right) .
\end{aligned}
$$

For $|z|<1$, that is $x \in \mathbb{C} /[-1,1]$, and $|a|<1$, we can deduce the following asymptotic formula for $p_{n}^{\alpha}(x)$ from (4.13):

$$
\begin{aligned}
\lim _{n \rightarrow \infty} & \left(\frac{z}{a}\right)^{n} p_{n}^{\alpha}(x) \\
= & \frac{\left(a b c d q^{2 \alpha-1}, a z, b z q^{\alpha}, c z q^{\alpha}, d z q^{\alpha}, b c d z q^{\alpha-1}\right)_{\infty}}{\left(a b c d q^{\alpha-1}, a b q^{\alpha}, a c q^{\alpha}, a d q^{\alpha}, b c d z q^{2 \alpha-1}, z^{2}\right)_{\infty}} \\
& \cdot{ }_{8} W_{7}\left(b c d z q^{2 \alpha-2} ; b c q^{\alpha-1}, b d q^{\alpha-1}, c d q^{\alpha-1}, q^{\alpha}, z q / a ; q, a z\right), \\
= & \frac{(a z)^{1-\alpha}\left(a z, b c q^{\alpha-1}, b d q^{\alpha-1}, c d q^{\alpha-1}\right)_{\infty}}{\left(1-a b c d q^{2 \alpha-2}\right)\left(a b q^{\alpha}, a c q^{\alpha}, a d q^{\alpha}, a b c d q^{\alpha-1}\right)_{\infty}} s_{\alpha-1}(z) .
\end{aligned}
$$

When we set $\alpha=0,(4.16)$ agrees with the asymptotic formula for the AskeyWilson polynomials $p_{n}(x ; a, b, c, d \mid q)$ obtained in Ismail and Wilson [17] and Rahman and Verma [24], when we set $\alpha=0$ in the above formula.

The asymptotic formula (4.16) enables us to evaluate the Stieltjes transform of the measure of orthogonality of the associated Askey-Wilson polynomials using (1.25). Let

$$
\int_{-\infty}^{\infty} d \mu(t ; \alpha, 1)=1, \quad \int_{-\infty}^{\infty} p_{n}^{\alpha}(t) p_{m}^{\alpha}(t) d \mu(t ; \alpha, 1)=0, \quad m \neq n .
$$

The reason we used 1 in $d \mu(t ; \alpha, 1)$ is that we will introduce a second family of associated Askey-Wilson polynomials and will denote the measure they are orthogonal with respect to, by $d \mu(t ; \alpha, 2)$. Applying (1.25) and (4.16) yields (4.18)

$$
\begin{aligned}
\int_{-\infty}^{\infty} & \frac{d \mu(t ; \alpha, 1)}{x-t} \\
= & \frac{2 z\left(1-b c d z q^{2 \alpha-1}\right)\left(1-b c d z q^{2 \alpha}\right)}{\left(1-b z q^{\alpha}\right)\left(1-c z q^{\alpha}\right)\left(1-d z q^{\alpha}\right)\left(1-b c d z q^{\alpha-1}\right)} \\
& \cdot \frac{{ }_{8} W_{7}\left(b c d z q^{2 \alpha} ; b c q^{\alpha}, b d q^{\alpha}, c d q^{\alpha}, q^{\alpha+1}, z q / a ; q, a z\right)}{{ }_{8} W_{7}\left(b c d z q^{2 \alpha-2} ; b c q^{\alpha-1}, b d q^{\alpha-1}, c d q^{\alpha-1}, q^{\alpha}, z q / a ; q, a z\right)}
\end{aligned}
$$

provided that $x$ is in the complex plane cut along $[-1,1]$.

This relation can also be obtained from the theory of continued fractions in the following manner. The functions $R_{n}(x), S_{n}(x), R_{n}(x):=r_{n+\alpha}(x)$, $S_{n}(x):=s_{n+\alpha}(x)$, are linearly independent solutions of the three term recurrence relation of the associated Askey-Wilson polynomials. When $|z|<1$, 
$S_{n}(x)$ is the minimal solution of the above-mentioned recurrence relation, since $S_{n}(x) / R_{n}(x) \rightarrow 0$ as $n \rightarrow \infty$. When $|z|=1$ it is clear from the explicit representations of $r_{n+\alpha}(x)$ and $s_{n+\alpha}(x)$ that both are oscillatory and their recurrence relation will have no minimal solution. We then apply Pincherle's Theorem, Jones and Thron [18], and conclude that the left-hand side of (4.18) must be a constant multiple of $S_{0}(x) / S_{-1}(x)$. The constant is then computed by letting $x \rightarrow \infty$ and noting that the left-hand side of (4.18) is $x^{-1}+O\left(x^{-2}\right)$ as $x \rightarrow \infty$.

From (4.18) one may invert the Stieltjes transform of $d \mu(t ; \alpha, 1)$ and find the measure $d \mu(t ; \alpha, 1)$ explicitly. The isolated points in the support of $d \mu(t ; \alpha, 1)$ coincide with the poles of the right-hand side of (4.18). Note that the ${ }_{8} W_{7}$ 's in (4.18) satisfy the same three term recurrence relation and it is possible to use this fact to prove that the ${ }_{8} W_{7}$ 's in (4.18) have no common zeros. We conjecture that the ${ }_{8} W_{7}$ in the denominator of (4.18) has no zeros for $|z|<1, \alpha \geq 0$, and $-q^{\alpha / 2}<a, b, c, d<q^{\alpha / 2}$. We have been able to prove this conjecture when $\alpha \geq 0$ and $-1<a, b, c, d<1$. The proof is given at the end of this section.

We now compute the absolutely continuous components of $d \mu(t ; \alpha, 1)$. Recall that the Perron-Stieltjes inversion formula implies

$$
\mu^{\prime}(t ; \alpha, 1)=\left[F\left(t-i 0^{+}\right)-F\left(t+i 0^{+}\right)\right] /(2 \pi i),
$$

where $F(x)$ denotes the right-hand side of (4.18). Since $F(z)$ is single valued for $|z|<1$ then one can see from (4.19) that $\mu^{\prime}$ is supported on $[-1,1]$. Observe that as we cross $[-1,1]$ from the upper or lower half planes the roles of $z, z^{-1}$ are interchanged. Note that

$$
F(x):=\int_{-\infty}^{\infty} \frac{d \mu(t ; \alpha, 1)}{x-t}=C s_{\alpha}(z) / s_{\alpha-1}(z),
$$

$C$ being a constant. From (4.19) and (4.20) it follows that

$$
\begin{aligned}
& \left.2 \pi i s_{\alpha-1}\left(e^{i \theta}\right)\right|^{2} \mu^{\prime}(\cos \theta ; \alpha, 1) \\
& \quad=C\left\{s_{\alpha}\left(e^{i \theta}\right) s_{\alpha-1}\left(e^{-i \theta}\right)-s_{\alpha}\left(e^{-i \theta}\right) s_{\alpha-1}\left(e^{i \theta}\right)\right\} .
\end{aligned}
$$

Using (1.13), (1.20) and the fact that the left-hand side of (4.18) is $x^{-1}+O\left(x^{-2}\right)$ as $x \rightarrow \infty$, we get

$$
a C=\frac{2\left(1-a b c d q^{2 \alpha-2}\right)\left(1-a b c d q^{2 \alpha-1}\right)}{\left(1-b c q^{\alpha-1}\right)\left(1-b d q^{\alpha-1}\right)\left(1-c d q^{\alpha-1}\right)\left(1-q^{\alpha}\right)},
$$

since $z \sim \frac{1}{2} x^{-1}$.

We now evaluate the right-hand side of (4.21). It readily follows from (1.11) that

$$
\begin{aligned}
& A_{\alpha}\left\{h_{\alpha}(\bar{z}) h_{\alpha+1}(z)-h_{\alpha}(z) h_{\alpha+1}(\bar{z})\right\} \\
& \quad=C_{\alpha}\left\{h_{\alpha-1}(\bar{z}) h_{\alpha}(z)-h_{\alpha-1}(z) h_{\alpha}(\bar{z})\right\} .
\end{aligned}
$$

Set

$$
U_{\alpha+1}(x):=h_{\alpha+1}(z) h_{\alpha}(\bar{z})-h_{\alpha+1}(\bar{z}) h_{\alpha}(z) \text {. }
$$


The above functional equation when expressed in term of $U_{\alpha}(x)$ is

$$
U_{\alpha}(x)=A_{\alpha} U_{\alpha+1}(x) / C_{\alpha},
$$

which, when iterated, leads to

$$
\begin{aligned}
U_{\alpha}(x)= & \frac{\left(a b q^{\alpha}, a c q^{\alpha}, a d q^{\alpha}, a b c d q^{\alpha-1}\right)_{\infty}}{\left(b c q^{\alpha-1}, b d q^{\alpha-1}, c d q^{\alpha-1}, q^{\alpha}\right)_{\infty}}\left(1-a b c d q^{2 \alpha-2}\right) \\
& \cdot \lim _{n \rightarrow \infty} a^{-2 n} U_{\alpha+n}(x)
\end{aligned}
$$

provided that the limit exists. When $h_{\alpha}(x)=s_{\alpha}(x)$, then in view of (1.13), we have

$$
\begin{aligned}
\lim _{n \rightarrow \infty} & a^{-2 n-2 \alpha} U_{n+\alpha}(x) \\
& =\lim _{n \rightarrow \infty}(a z)^{-n-\alpha}(a \bar{z})^{-n-\alpha}\left\{s_{n+\alpha}(z) s_{n+\alpha-1}(\bar{z})-s_{n+\alpha}(\bar{z}) s_{n+\alpha-1}(z)\right\} \\
& =(a \bar{z})^{-1} \frac{\left(q z^{2}, q \bar{z}^{2}\right)_{\infty}}{(a z, a \bar{z})_{\infty}}-(a z)^{-1} \frac{\left(q z^{2}, q \bar{z}^{2}\right)_{\infty}}{(a z, a \bar{z})_{\infty}} \\
& =\frac{z-\bar{z}}{a|z|^{2}}\left|\frac{\left(q z^{2}\right)_{\infty}}{(a z)_{\infty}}\right|^{2} .
\end{aligned}
$$

This implies that

$$
\lim _{n \rightarrow \infty} a^{-2 n} U_{n+\alpha}(x)=-a^{2 \alpha-1}(2 i \sin \theta)\left|\frac{\left(q e^{2 i \theta}\right)_{\infty}}{\left(a e^{i \theta}\right)_{\infty}}\right|^{2}
$$

where $z=e^{-i \theta}, x=\cos \theta$. We now combine (4.28) with (4.21), (4.22) and (4.26), and find the absolutely continuous component of the spectral measure to have the form

(4.29)

$$
\begin{aligned}
& \frac{d \mu(\cos \theta ; \alpha, 1)}{d \theta} \\
& =\frac{\left(a b q^{\alpha}, a c q^{\alpha}, a d q^{\alpha}, b c q^{\alpha}, b d q^{\alpha}, c d q^{\alpha}, a b c d q^{\alpha-1}, q^{\alpha+1}\right)_{\infty}}{2 \pi\left(a b c d q^{2 \alpha-1}, a b c d q^{2 \alpha}\right)_{\infty}} \\
& \quad \cdot\left|\frac{\left(e^{2 i \theta}, b c d q^{2 \alpha-1} e^{i \theta}\right)_{\infty}}{\left(a e^{i \theta}, b q^{\alpha} e^{i \theta}, c q^{\alpha} e^{i \theta}, d q^{\alpha} e^{i \theta}, b c d q^{\alpha-1} e^{i \theta}\right)_{\infty}}\right|^{2} \\
& \quad \cdot\left|{ }_{8} W_{7}\left(b c d q^{2 \alpha-2} e^{i \theta} ; b c q^{\alpha-1}, b d q^{\alpha-1}, c d q^{\alpha-1}, q^{\alpha}, q e^{i \theta} / a ; q, a e^{i \theta}\right)\right|^{-2} .
\end{aligned}
$$

The above weight function $d \mu / d \theta$ is symmetric in the parameters $a, b, c, d$ but this symmetry is not manifested in the expression on the right-hand side of (4.29). In order to exhibit the symmetry in $a, b, c, d$ we apply the transfor- 
mation formula [13, (2.10.1)]

$$
\begin{aligned}
{ }_{8} W_{7}\left(a ; b, c, d, e, f ; q, a^{2} q^{2} / b c d e f\right) \\
=\frac{\left(a q, a q / e f, a^{2} q^{2} / b c d e, a^{2} q^{2} / b c d f\right)_{\infty}}{\left(a q / e, a q / f, a^{2} q^{2} / b c d, a^{2} q^{2} / b c d e f\right)_{\infty}} \\
\quad \cdot{ }_{8} W_{7}\left(a^{2} q / b c d ; a q / b c, a q / b d, a q / c d, e, f ; q, a q / e f\right),
\end{aligned}
$$

and establish the symmetric representation

(4.31)

$$
\begin{aligned}
& \frac{d \mu(\cos \theta ; \alpha, 1)}{d \theta} \\
& =\frac{\left(a b q^{\alpha}, a c q^{\alpha}, a d q^{\alpha}, b c q^{\alpha}, b d q^{\alpha}, c d q^{\alpha}, q^{\alpha+1}\right)_{\infty}}{2 \pi\left(a b c d q^{2 \alpha}\right)_{\infty}\left(1-a b c d q^{\alpha-2}\right)\left(a b c d q^{\alpha-2}\right)_{\infty}}\left(1-a b c d q^{2 \alpha-2}\right) \\
& \cdot\left(a b c d q^{2 \alpha-2}\right)_{\infty}\left|\frac{\left(e^{2 i \theta}, q^{\alpha+1} e^{2 i \theta}\right)_{\infty}}{\left(a q^{\alpha} e^{i \theta}, b q^{\alpha} e^{i \theta}, c q^{\alpha} e^{i \theta}, d q^{\alpha} e^{i \theta}, q e^{2 i \theta}\right)_{\infty}}\right|^{2} \\
& \left.\left.\cdot\right|_{8} W_{7}\left(q^{\alpha} e^{2 i \theta} ; q e^{i \theta} / a, q e^{i \theta} / b, q e^{i \theta} / c, q e^{i \theta} / d, q^{\alpha} ; q, a b c d q^{\alpha-2}\right)\right|^{-2} .
\end{aligned}
$$

We conclude this section by showing that the discrete spectrum is empty. Since $A_{n+\alpha}, B_{n+\alpha}$ and $C_{n+\alpha}$ of (1.11) are bounded, the support of $d \mu(x ; \alpha, 1)$ will be bounded, Chihara [12, Theorem IV 2.2], and the moment problem is determined. Therefore a point $x=\zeta$ supports a discrete mass if and only if

$$
\sum_{n=0}^{\infty}\left[p_{n}^{\alpha}(\zeta)\right]^{2} / \xi_{n}
$$

converges, Shohat and Tamarkin [26], where $\xi_{n}$ is as in (1.7), (1.18) and (1.19). To show that $x= \pm 1$ do not support masses we need to show that the series (4.32) diverges at $\zeta= \pm 1$. To determine the asymptotic behavior of $p_{n}^{\alpha}( \pm 1)$ we use the Birkhoff-Trjitzinsky theory of difference equations, Wimp [32]. We assume

$$
p_{n}^{\alpha}( \pm 1) \approx r^{n} n^{k}\left\{1+\gamma n^{-1}+\delta n^{-2}+O\left(n^{-3}\right)\right\}
$$

then substitute for $p_{n}^{\alpha}( \pm 1)$ from (4.33) in (4.14) and equate coefficients. The result is $r= \pm a$ at $x= \pm 1$ and the possible values of $k$ are $k=0,1$. This shows that there are two linearly independent solutions of (4.14) at $x=1$ and as $n \rightarrow \infty$ they are $O\left(a^{n}\right)$ and $O\left(n a^{n}\right)$. The solutions at $x=-1$ have the same orders of magnitude. In the case under consideration, $\xi_{n} \approx C a^{2 n}$, for some $C \neq 0$, and the series in (4.32) will diverge at $\zeta= \pm 1$.

It now remains to show that there are no masses outside $[-1,1]$, i.e. the true interval of orthogonality is $[-1,1]$, Chihara [12, p. 29]. Recall that for $\left\{p_{n}(x)\right\}$ of $(1.7)$, an interval $[r, s]$ contains the true interval of orthogonality if only if $r<b_{n}<s, n \geq 0$, and $\left\{\beta_{n}(x)\right\}_{1}^{\infty}$ is a chain sequence at $x=r$ and $x=s$, where

$$
\beta_{n}(x)=a_{n-1} c_{n} /\left[\left(x-b_{n}\right)\left(x-b_{n-1}\right)\right], \quad n>0,
$$


[12, p. 108]. In our case of interest, namely, $a_{n}=A_{n+\alpha}, b_{n}=B_{n+\alpha}, c_{n}=C_{n+\alpha}$, denote $\beta_{n}(x)$ by $\beta_{n}(x ; \alpha)$. The true interval of orthogonality of the AskeyWilson polynomials is $[-1,1]$, thus $B_{n} \in(-1,1)$ and $\left\{\beta_{n}( \pm 1 ; 0)\right\}$ are chain sequences. When $\alpha=k, \beta_{n}(x ; k)=\beta_{n+k}(x ; 0)$ so $B_{n+k} \in(-1,1)$ and $\left\{\beta_{n}(x ; k)\right\}_{1}^{\infty}$ is a chain sequence when $x= \pm 1$. Therefore,

$$
\int_{-1}^{1} \mu^{\prime}(x ; \alpha, 1) d x=1, \quad \alpha=0,1,2, \ldots .
$$

Since the left-hand side of (4.35) is analytic in $w=q^{\alpha}$ for $|w|<1$, then (4.35) will continue to hold for $\alpha \geq 0$, by the identity theorem of analytic functions. On the other hand, $d \mu$ is a positive measure normalized to have total mass 1 , so it cannot have a discrete component. This proves the first part of Theorem 3.

Bustoz and Ismail [10] studied the associated continuous $q$-ultraspherical polynomials $\left\{C_{n}^{\alpha}(x ; \beta \mid q)\right\}$, which correspond to $a=-d=\sqrt{q}, b=-c=$ $\beta \sqrt{q}, \gamma=q^{\alpha}$. They proved that the discrete spectrum is empty if $0<\beta<1$, $0<q<1,0 \leq \alpha<1$, or if $q^{2}<\beta<1,0<q<1,-1<\alpha<0$. Observe that our result concerning the absence of a discrete spectrum shows that the discrete spectrum of the associated continuous $q$-ultraspherical polynomials is empty when $-1<\beta<1,0<q<1$, and $\alpha \geq 0$. This extends some of the results in [10] to much wider range of the parameters.

\section{A SECOND SYSTEM OF ASSOCIATED ASKEY-WILSON POLYNOMIALS}

Let us now consider the linear combination:

$$
q_{n}^{\alpha}(x)=q_{n}^{\alpha}(x ; a, b, c, d \mid q)=L_{\alpha}^{\prime} r_{\alpha+n}(x)+M_{\alpha}^{\prime} s_{\alpha+n}(z),
$$

subject to the conditions

$$
q_{0}^{\alpha}(x) \equiv 1 \quad \text { and } \quad q_{1}^{\alpha}(x)=1+A_{\alpha}^{-1}\left(z+z^{-1}-a-a^{-1}\right) .
$$

Use of (1.11) then gives:

$$
\begin{aligned}
L_{\alpha}^{\prime} & =-W_{\alpha}^{-1} A_{\alpha}^{-1} C_{\alpha}\left(s_{\alpha}(z)-s_{\alpha-1}(z)\right), \\
M_{\alpha}^{\prime} & =W_{\alpha}^{-1} A_{\alpha}^{-1} C_{\alpha}\left(r_{\alpha}(z)-r_{\alpha-1}(z)\right),
\end{aligned}
$$

where,

$$
\begin{aligned}
W_{\alpha}= & r_{\alpha+1}(z) s_{\alpha}(z)-r_{\alpha}(z) s_{\alpha+1}(z)=A_{\alpha+1} W_{\alpha+1} / C_{\alpha+1} \\
= & \frac{\left(1-a b q^{\alpha+1}\right)\left(1-a c q^{\alpha+1}\right)\left(1-a d q^{\alpha+1}\right)\left(1-a b c d q^{\alpha}\right)\left(1-a b c d q^{2 \alpha}\right)}{\left(1-b c q^{\alpha}\right)\left(1-b d q^{\alpha}\right)\left(1-c d q^{\alpha}\right)\left(1-q^{\alpha+1}\right)\left(1-a b c d q^{2 \alpha+2}\right)} \\
& \cdot a^{-2} \cdot W_{\alpha+1} \cdot
\end{aligned}
$$

As in the previous section we can solve this functional equation by iteration 
and obtain

$$
\begin{aligned}
W_{\alpha}= & \frac{a^{2 \alpha+1}}{z}\left(1-a b c d q^{2 \alpha}\right) \\
& \cdot \frac{\left(a b q^{\alpha+1}, a c q^{\alpha+1}, a d q^{\alpha+1}, a b c d q^{\alpha}, b z, c z, d z, b c d / z\right)_{\infty}}{\left(b c q^{\alpha}, b d q^{\alpha}, c d q^{\alpha}, q^{\alpha+1}, b c, b d, c d, a z\right)_{\infty}} .
\end{aligned}
$$

Hence we get

$$
q_{n}^{\alpha}(x)=W_{\alpha-1}^{-1}\left\{\left[r_{\alpha}(z)-r_{\alpha-1}(z)\right] s_{\alpha+n}(z)-\left[s_{\alpha}(z)-s_{\alpha-1}(z)\right] r_{\alpha+n}(z)\right\} .
$$

As for the asymptotics it is clear that for $|z|<1$,

$$
\begin{aligned}
\lim _{n \rightarrow \infty} & \left(\frac{z}{a}\right)^{n} q_{n}^{\alpha}(x) \\
& =W_{\alpha-1}^{-1}\left[s_{\alpha-1}(z)-s_{\alpha}(z)\right]\left(\frac{a}{z}\right)^{\alpha} \frac{(b c d / z, b z, c z, d z)_{\infty}}{\left(b c, b d, c d, z^{2}\right)_{\infty}} \\
& =(a z)^{1-\alpha} \frac{\left(b c q^{\alpha-1}, b d q^{\alpha-1}, c d q^{\alpha-1}, q^{\alpha}, a z\right)_{\infty}}{\left(a b q^{\alpha}, a c q^{\alpha}, a d q^{\alpha}, a b c d q^{\alpha-1}, z^{2}\right)_{\infty}} \frac{\left.[z)-s_{\alpha}(z)\right]}{1-a b c d q^{2 \alpha-2}} .
\end{aligned}
$$

We will now show that $s_{\alpha-1}(z)-s_{\alpha}(z)$ can be expressed as a multiple of an ${ }_{8} W_{7}$ function, namely,

$$
\begin{gathered}
s_{\alpha-1}(z)-s_{\alpha}(z)=\frac{\left(a b c d q^{2 \alpha-2}, b z q^{\alpha}, c z q^{\alpha}, d z q^{\alpha}, b c d z q^{\alpha-1}\right)_{\infty}}{\left(b c q^{\alpha-1}, b d q^{\alpha-1}, c d q^{\alpha-1}, q^{\alpha}, b c d z q^{2 \alpha-1}\right)_{\infty}}(a z)^{\alpha-1} \\
\cdot{ }_{8} W_{7}\left(b c d z q^{2 \alpha-2} ; b c q^{\alpha-1}, b d q^{\alpha-1}, c d q^{\alpha-1}, q^{\alpha}, z / a ; q, a q z\right) .
\end{gathered}
$$

After we prove (5.8) we will show that $\left\{q_{n}^{\alpha}(x)\right\}$ are orthogonal with respect to an absolutely continuous measure $d \mu(x ; \alpha, 2)$ supported on $[-1,1]$. We will also find $\mu^{\prime}(x ; \alpha, 2)$ explicitly.

To prove (5.8) observe that (1.13) implies

$$
\begin{aligned}
s_{\alpha-1}(z) & -s_{\alpha}(z) \\
= & \frac{\left(a b c d q^{2 \alpha-2}, b z q^{\alpha}, c z q^{\alpha}, d z q^{\alpha}, b c d z q^{\alpha-1}\right)_{\infty}}{\left(b c q^{\alpha-1}, b d q^{\alpha-1}, c d q^{\alpha-1}, q^{\alpha}, b c d z q^{2 \alpha-1}\right)_{\infty}}(a z)^{\alpha-1} \\
+ & \sum_{k=0}^{\infty} \frac{\left(a b c d q^{2 \alpha+k-1}, b z q^{\alpha+1+k}, d z q^{\alpha+1+k}, b c d z q^{\alpha+k}\right)_{\infty}}{\left(b c q^{\alpha+k}, b d q^{\alpha+k}, c d q^{\alpha+k}, q^{\alpha+1+k}, b c d z q^{2 \alpha+k-1}\right)_{\infty}} \\
& \cdot\left(1-b c d z q^{2 \alpha+2 k}\right) \frac{(z q / a)_{k+1}(a z)^{\alpha+k}}{(q)_{k+1}} \\
& -\sum_{k=0}^{\infty} \frac{\left(a b c d q^{2 \alpha+k}, b z q^{\alpha+1+k}, c z q^{\alpha+1+k}, d z q^{\alpha+1+k}, b c d z q^{\alpha+k}\right)_{\infty}}{\left(b c q^{\alpha+k}, b d q^{\alpha+k}, c d q^{\alpha+k}, q^{\alpha+1+k}, b c d z q^{2 \alpha+k}\right)_{\infty}} \\
& \cdot\left(1-b c d z q^{2 \alpha+2 k}\right) \frac{(z q / a)_{k}(a z)^{\alpha+k}}{(q)_{k}} .
\end{aligned}
$$


Since

$$
\begin{aligned}
\frac{\left(a b c d q^{2 \alpha+k-1}\right)_{\infty}(z q / a)_{k+1}}{\left(b c d z q^{2 \alpha+k-1}\right)_{\infty}(q)_{k+1}}-\frac{\left(a b c d q^{2 \alpha+k}\right)_{\infty}(z q / a)_{k}}{\left(b c d z q^{2 \alpha+k}\right)_{\infty}(q)_{k}} & \left(a b c d q^{2 \alpha+k}\right)_{\infty}(z q / a)_{k} \\
= & \frac{\left(b c d z q^{2 \alpha+k-1}\right)_{\infty}(q)_{k+1}}{\left(b c d z q^{2 \alpha+k-1}\right)_{\infty}(q)_{k+1}} \\
& \cdot\left\{\left(1-a b c d q^{2 \alpha+k-1}\right)\left(1-z q^{k+1} / \alpha\right)-\left(1-q^{k+1}\right)\left(1-b c d z q^{2 \alpha+k-1}\right)\right\} \\
= & \frac{q^{k+1}\left(1-a b c d q^{2 \alpha-2}\right)\left(a b c d q^{2 \alpha+k}\right)_{\infty+1}(z / a)_{k+1}}{(1-a)}
\end{aligned}
$$

we find that

$$
\begin{aligned}
& s_{\alpha-1}(z)-s_{\alpha}(z) \\
& =\frac{\left(a b c d q^{2 \alpha-2}, b z q^{\alpha}, c z q^{\alpha}, d z q^{\alpha}, b c d z q^{\alpha-1}\right)_{\infty}}{\left(b c q^{\alpha-1}, b d q^{\alpha-1}, c d q^{\alpha-1}, q^{\alpha}, b c d z q^{2 \alpha-1}\right)_{\infty}}(a z)^{\alpha-1} \\
& \cdot\left\{1+\sum_{k=1}^{\infty} \frac{\left(1-b c d z q^{2 \alpha+2 k-2}\right)\left(b c d z q^{2 \alpha-2}, b c q^{\alpha-1}, b d q^{\alpha-1}, c d q^{\alpha-1}, q^{\alpha}, z / a\right)_{k}}{\left(1-b c d z q^{2 \alpha-2}\right)\left(q, b z q^{\alpha}, c z q^{\alpha}, d z q^{\alpha}, b c d z q^{\alpha-1}, a b c d q^{2 \alpha-1}\right)_{k}}\right. \\
& \left.\cdot(a q z)^{k}\right\} \text {, }
\end{aligned}
$$

which immediately leads to $(5.8)$.

We now proceed to find $d \mu(x ; \alpha, 2)$ explicitly. It is easy to see that $\left(q_{n}^{\alpha}(x)\right)^{*}$ $=\left(p_{n}^{\alpha}(x)\right)^{*}$, hence

$$
\frac{1}{2} A_{\alpha} \int_{-\infty}^{\infty} \frac{d \mu(t ; \alpha, 2)}{x-t}=\lim _{n \rightarrow \infty} p_{n-1}^{\alpha+1}(x) / q_{n}^{\alpha}(x),
$$

which follows from (1.23), (1.24) and (1.25). Using (4.16) and (5.7) we find that

$$
\int_{-\infty}^{\infty} \frac{d \mu(t ; \alpha, 2)}{x-t}=\frac{D_{\alpha} s_{\alpha}(z)}{s_{\alpha-1}(z)-s_{\alpha}(z)}
$$

where,

$$
\begin{aligned}
D_{\alpha} & =2 / C_{\alpha} \\
& =\frac{2\left(1-a b c d q^{2 \alpha-1}\right)\left(1-a b c d q^{2 \alpha-2}\right)}{a\left(1-b c q^{\alpha-1}\right)\left(1-b d q^{\alpha-1}\right)\left(1-c d q^{\alpha-1}\right)\left(1-q^{\alpha}\right)} .
\end{aligned}
$$

Now the Perron-Stieltjes inversion formula, as in (4.19), implies

$$
\begin{aligned}
& 2 \pi i \mu^{\prime}(x ; \alpha, 2) \\
& =D_{\alpha}\left\{\frac{s_{\alpha}\left(e^{-i \theta}\right)}{s_{\alpha-1}\left(e^{-i \theta}\right)-s_{\alpha}\left(e^{-i \theta}\right)}-\frac{s_{\alpha}\left(e^{i \theta}\right)}{s_{\alpha-1}\left(e^{i \theta}\right)-s_{\alpha}\left(e^{i \theta}\right)}\right\} \\
& =\frac{D_{\alpha}\left\{s_{\alpha}\left(e^{-i \theta}\right) s_{\alpha-1}\left(e^{i \theta}\right)-s_{\alpha}\left(e^{i \theta}\right) s_{\alpha-1}\left(e^{-i \theta}\right)\right\}}{\left|s_{\alpha-1}\left(e^{i \theta}\right)-s_{\alpha}\left(e^{i \theta}\right)\right|^{2}},
\end{aligned}
$$


with $x=\cos \theta$, as usual. Recall that in $\S 4$ we proved that

$$
\begin{aligned}
U_{\alpha}(x)= & s_{\alpha}\left(e^{-i \theta}\right) s_{\alpha-1}\left(e^{i \theta}\right)-s_{\alpha}\left(e^{i \theta}\right) s_{\alpha-1}\left(e^{-i \theta}\right) \\
= & -\frac{\left(a b q^{\alpha}, a c q^{\alpha}, a d q^{\alpha}, a b c d q^{\alpha-1}\right)_{\infty}}{\left(b c q^{\alpha-1}, b d q^{\alpha-1}, c d q^{\alpha-1}, q^{\alpha}\right)_{\infty}}\left(1-a b c d q^{2 \alpha-2}\right) a^{2 \alpha-1} \\
& \cdot 2 i \sin \theta\left|\frac{\left(q e^{2 i \theta}\right)_{\infty}}{\left(a e^{i \theta}\right)_{\infty}}\right|^{2} .
\end{aligned}
$$

Applying the transformation (4.30) on the ${ }_{8} W_{7}$ series on the right-hand side of (5.8) we then obtain from (5.12), (5.13) and (5.14) that

$$
\begin{aligned}
\frac{d \mu(\cos \theta ; \alpha, 2)}{d \theta} & \frac{\left(a b q^{\alpha}, a c q^{\alpha}, a d q^{\alpha}, b c q^{\alpha}, b d q^{\alpha}, c d q^{\alpha}, q^{\alpha+1}\right)_{\infty}}{2 \pi\left(a b c d q^{2 \alpha}\right)_{\infty}} \\
= & \cdot \frac{\left(a b c d q^{2 \alpha-1}\right)_{\infty}}{\left(a b c d q^{\alpha-1}\right)_{\infty}} \frac{\left(1-2 a x q^{\alpha}+a^{2} q^{2 \alpha}\right)}{\left(1-2 a x+a^{2}\right)} \\
& \cdot \frac{\left|\left(e^{2 i \theta}, q^{\alpha+1} e^{2 i \theta}\right)\right|_{\infty}^{2}}{\left|\left(a q^{\alpha} e^{i \theta}, b q^{\alpha} e^{i \theta}, c q^{\alpha} e^{i \theta}, d q^{\alpha} e^{i \theta}, q e^{2 i \theta}\right)\right|_{\infty}^{2}} \\
& \cdot\left|{ }_{8} W_{7}\left(q^{\alpha} e^{2 i \theta} ; e^{i \theta} / a, q e^{i \theta} / b, q e^{i \theta} / c, q e^{i \theta} / d, q^{\alpha} ; q, a b c d q^{\alpha-1}\right)\right|^{-2}
\end{aligned}
$$

Finally we prove that $d \mu(x ; \alpha, 2)$ has at most one discrete mass if $0<q<1$, $\alpha \geq 0,-1<a, b, c, d<1$. Nevai's theorem, Theorem 1 , shows that $(-1,1)$ is free of discrete masses. It can be shown, as was done for $\left\{p_{n}^{\alpha}(x)\right\}$, that $x= \pm 1$ do not support discrete masses. If the zeros of polynomials are arranged in increasing order then the $j$ th zero of $q_{n}^{\alpha}(x)$ lies between the $j$ th zero of $p_{n}^{\alpha}(x)$ and the $j$ th zero of $p_{n-1}^{\alpha+1}(x)$, since $\left(p_{n}^{\alpha}(x)\right)^{*}$ is a constant multiple of $p_{n-1}^{\alpha+1}(x)$. Thus, with at most one exception the zeros of $q_{n}^{\alpha}(x)$ lie in $(-1,1)$ for all $n$ in the appropriate range of the parameters. This proves the part of Theorem 3 concerning the ${ }_{8} W_{7}$ in $(5.15)$.

Finally we note that if $a>1$ or $a<-1$, the $d \mu(x ; \alpha, 2)$ will have a mass at $\left(a+a^{-1}\right) / 2$. This is the case since $q_{n}^{\alpha}\left(\left(a+a^{-1}\right) / 2\right)=1$, which can be proved by induction, implies the convergence of the series $\sum_{0}^{\infty}\left[q_{n}^{\alpha}\left(\left(a+a^{-1}\right) / 2\right)\right]^{2} / \xi_{n}$.

\section{Proof of (4.15)}

We shall now give an inductive proof of equation (4.15). Recall that $p_{n}^{\alpha}(x ; a, b, c, d \mid q)$ satisfies the recurrence relation

$$
\begin{aligned}
& -a^{-1}(1-a z)(1-a / z) p_{n}^{\alpha}(x ; a, b, c, d \mid q) \\
& \quad=A_{\alpha+n}(a, b, c, d)\left\{p_{n+1}^{\alpha}(x ; a, b, c, d \mid q)-p_{n}^{\alpha}(x ; a, b, c, d \mid q)\right\} \\
& \quad-C_{\alpha+n}(a, b, c, d)\left\{p_{n}^{\alpha}(x ; a, b, c, d \mid q)-p_{n-1}^{\alpha}(x ; a, b, c, d \mid q)\right\}
\end{aligned}
$$


where $p_{-1}^{\alpha}(x) \equiv 0, p_{0}^{\alpha}(x)=1$, and the coefficients are defined in (1.14) and (1.15). Using (6.1) it can be easily verified that

$$
p_{n}^{\alpha}(x ; a, b, c, d \mid q)=\frac{\left(b d q^{\alpha}, c d q^{\alpha}\right)_{n}}{\left(a b q^{\alpha}, a c q^{\alpha}\right)_{n}}(a / d)^{n} p_{n}^{\alpha}(x ; d, b, c, a \mid q) \text {. }
$$

The proof of (4.15) depends heavily on the use of the following transformation of Sears [26]:

$$
\begin{aligned}
{ }_{4} \phi_{3}\left[\begin{array}{l}
q^{-n}, a, b, c \\
d, e, f
\end{array} q, q\right] \\
\quad=\frac{(d / a, e / a)_{n}}{(d, e)_{n}} \phi_{4}\left[\begin{array}{c}
q^{-n}, a, f / b, f / c \\
f, a q^{1-n} / d, a q^{1-n} / e
\end{array} ; q, q\right],
\end{aligned}
$$

where $d e f q^{n}=a b c q$, (see for example, equation (2.10.4) in Gasper and Rahman [13]), Watson's formula,

$$
\begin{aligned}
& { }_{8} W_{7}\left(d e / c q ; a, b, d / c, e / c, q^{-n} ; q, d e q^{n} / a b\right) \\
& \quad=\frac{(d e / c, d e / a b c)}{(d e / a c, d e / b c)_{n}}{ }_{4} \phi_{3}\left[\begin{array}{c}
q^{-n}, a, b, c \\
d, e, a b c q^{1-n} / d e
\end{array} ; q, q\right]
\end{aligned}
$$

(equation (2.5.1) of [13]), and the expansion,

$$
\begin{aligned}
{ }_{10} W_{9} & \left(a ; b, c, d, e, f, g, q^{-m} ; q, a^{3} q^{m+3} / b c d e f g\right) \\
= & \frac{(a q, a q / f g)_{m}}{(a q / f, a q / g)_{m}} \sum_{j=0}^{m} \frac{\left(q^{-m}, f, g, a q / d e\right)_{j}}{\left(q, a q / d, a q / e, f g q^{-m} / a\right)_{j}} q^{j} \\
& \cdot{ }_{4} \phi_{3}\left[\begin{array}{c}
q^{-j}, d, e, a q / b c \\
a q / b, a q / c, d e q^{-j} / a
\end{array} ; q, q\right],
\end{aligned}
$$

see Exercise 2.20 in [13].

Assuming (4.15) to be true one can verify that

$$
\begin{aligned}
p_{n+1}^{\alpha}(x) & -p_{n}^{\alpha}(x) \\
= & \frac{a\left(z+z^{-1}-a-a^{-1}\right)\left(1-a b c d q^{2 \alpha-1}\right)\left(1-a b c d q^{2 \alpha+2 n}\right) q^{-n}}{\left(1-a b q^{\alpha}\right)\left(1-a c q^{\alpha}\right)\left(1-a d q^{\alpha}\right)\left(1-a b c d q^{\alpha-1}\right)} \\
& \cdot \sum_{k=0}^{n} \frac{\left(q^{-n}, a b c d q^{2 \alpha+n}, a b c d q^{2 \alpha}, a q z, a q / z\right)_{k}}{\left(q, a b q^{\alpha+1}, a c q^{\alpha+1}, a d q^{\alpha+1}, a b c d q^{\alpha}\right)_{k}} q^{\alpha-1}, q^{\alpha}, b c q^{\alpha-1}, b d q^{\alpha-1}, c d q^{\alpha-1}, \\
& \left.\cdot{ }_{10}^{k+2}, a b c d q^{2 \alpha+n+k}, q^{k-n} ; q, q q^{2}\right) .
\end{aligned}
$$

By (6.5), the ${ }_{10} W_{9}$ series in (6.6) can be expanded as

$$
\begin{aligned}
& \frac{\left(a b c d q^{2 \alpha+k}, a d q^{k+1}\right)_{n-k}}{\left(a b c d q^{\alpha+k}, a d q^{\alpha+k+1}\right)_{n-k}} \sum_{j=0}^{n-k} \frac{\left(q^{k-n}, q^{\alpha}, b c q^{\alpha-1}, a q^{k+2} / d\right)_{j}}{\left(q, a b q^{\alpha+k+1}, a c q^{\alpha+k+1}, q^{-n} / a d\right)_{j}} q^{j} \\
& \cdot{ }_{4} \phi_{3}\left[\begin{array}{c}
q^{-j}, b d q^{\alpha-1}, c d q^{\alpha-1}, q^{-k-n-2} \\
a b c d q^{2 \alpha-2}, q^{-n}, d q^{-k-j-1} / a
\end{array} ; q, q\right]
\end{aligned}
$$


and the ${ }_{4} \phi_{3}$ series is, in turn, transformed to

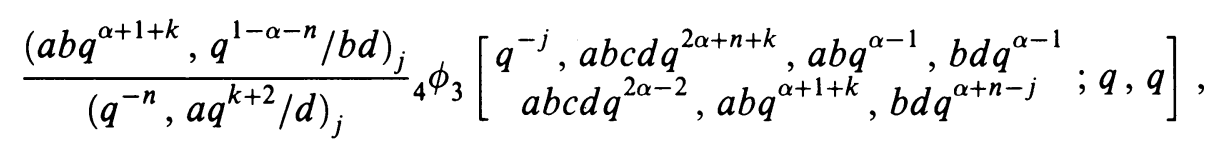

by (6.3). Denoting the series on the right-hand side of (6.6) by $S_{n}$, we then find that

$$
\begin{aligned}
S_{n}= & \frac{\left(a b c d q^{2 \alpha}, a d q\right)_{n}}{\left(a b c d q^{\alpha}, a d q^{\alpha+1}\right)_{n}} \sum_{k=0}^{n} \frac{\left(q^{-n}, a b c d q^{2 \alpha+n}, a q z, a q / z\right)_{k}}{\left(q, a d q, a b q^{\alpha+1}, a c q^{\alpha+1}\right)_{k}} q^{k} \\
& \cdot \sum_{j=0}^{n-k} \frac{\left(q^{k-n}, q^{\alpha}, b c q^{\alpha-1}, q^{1-\alpha-n} / b d\right)_{j}}{\left(q, q^{-n}, q^{-n} / a d, a c q^{\alpha+k+1}\right)_{j}} q^{j} \\
& \cdot \sum_{i=0}^{j} \frac{\left(q^{-j}, a b c d q^{2 \alpha+n+k}, a b q^{\alpha-1}, b d q^{\alpha-1}\right)_{i}}{\left(q, a b c d q^{2 \alpha-2}, a b q^{\alpha+1+k}, b d q^{\alpha+n-j}\right)_{i}} q^{i} \\
= & \frac{\left(a b c d q^{2 \alpha}, a d q\right)_{n}}{\left(a b c d q^{\alpha}, a d q^{\alpha+1}\right)_{n}} \sum_{j=0}^{n} \frac{\left(q^{-n}, q^{\alpha}, b c q^{\alpha-1}, q^{1-\alpha-n} / b d\right)_{j}}{\left(q, q^{-n}, q^{-n} / a d, a c q^{\alpha+1}\right)_{j}} q^{j} \\
& \cdot \sum_{i=0}^{j} \frac{\left(q^{-j}, a b c d q^{2 \alpha+n}, a b q^{\alpha-1}, b d q^{\alpha-1}\right)_{i}}{\left(q, a b c d q^{2 \alpha-2}, a b q^{\alpha+1}, b d q^{\alpha+n-j}\right)_{i}} \\
& \cdot{ }_{4} \phi_{3}\left[\begin{array}{c}
q^{j-n}, a b c d q^{2 \alpha+n+i}, a q z, a q / z \\
a d q, a b q^{\alpha+1+i}, a c q^{\alpha+1+j}
\end{array} ;, q\right] .
\end{aligned}
$$

Applying (6.3) to the last ${ }_{4} \phi_{3}$ series in (6.7) and simplifying the coefficients we get

$$
\begin{aligned}
&{ }_{4} \phi_{3}\left[\begin{array}{c}
q^{j-n}, a b c d q^{2 \alpha+n+i}, a q z, a q / z \\
a d q, a b q^{\alpha+1}, a c q^{\alpha+1+j}
\end{array}, q, q\right] \\
&=\left(\frac{a q}{d}\right)^{n} \frac{\left(c d q^{\alpha}, b d q^{\alpha}\right)_{n}}{\left(a b q^{\alpha+1}, a c q^{\alpha+1}\right)_{n}} \frac{\left(a b q^{\alpha+1}, b d q^{\alpha+n-j}\right)_{i}}{\left(c d q^{\alpha}, q^{1-\alpha-n} / b d\right)_{j}} \\
& \cdot \frac{\left(a c q^{\alpha+1}, q^{-\alpha-n} / a b\right)_{j}}{\left(b d q^{\alpha}, a b q^{\alpha+1+n-j}\right)_{i}} \cdot{ }_{4} \phi_{3}\left[\begin{array}{c}
q^{j-n}, a b c d q^{2 \alpha+n+i}, d z, d / z \\
a d q, c d q^{\alpha+j}, b d q^{\alpha+i}
\end{array} q, q\right] .
\end{aligned}
$$

Substituting (6.8) into (6.7) and interchanging the order of summation 
we obtain

$$
\begin{aligned}
S_{n}= & \frac{\left(b d q^{\alpha}, c d q^{\alpha}, a b c d q^{2 \alpha}, a d q\right)_{n}}{\left(a b q^{\alpha+1}, a c q^{\alpha+1}, a d q^{\alpha+1}, a b c d q^{\alpha}\right)_{n}}(a q / d)^{n} \\
& \cdot \sum_{k=0}^{n} \frac{\left(q^{-n}, a b c d q^{2 \alpha+n}, d z, d / z\right)_{k}}{\left(q, a d q, b d q^{\alpha}, c d q^{\alpha}\right)_{k}} q^{k} \\
& \cdot \sum_{j=0}^{n-k} \frac{\left(q^{k-n}, q^{\alpha}, b c q^{\alpha-1}, q^{-\alpha-n} / a b\right)_{j}}{\left(q, q^{-n}, q^{-n} / a d, c d q^{\alpha+k}\right)_{j}} q^{j} \\
& \cdot{ }_{4} \phi_{3}\left[\begin{array}{c}
q^{-j}, a b c d q^{2 \alpha+n+k}, a b q^{\alpha-1}, b d q^{\alpha-1} \\
\left.a b c d q^{2 \alpha-2}, b d q^{\alpha+k}, a b q^{\alpha+1+n-j} ; q, q\right]
\end{array}\right.
\end{aligned}
$$

Incredible as it may seem we now have to transform the ${ }_{4} \phi_{3}$ series in (6.9) into an ${ }_{8} W_{7}$ series by (6.4). Thus we have

$$
\begin{aligned}
{ }_{4} \phi_{3}\left[\begin{array}{c}
q^{-j}, a b c d q^{2 \alpha+n+k}, a b q^{\alpha-1}, b d q^{\alpha-1} \\
\left.a b c d q^{2 \alpha-2}, b d q^{\alpha+k}, a b q^{\alpha+1+n-j} ; q, q\right]
\end{array}\right] \\
=\frac{\left(c d q^{\alpha+k}, q^{-n-1}\right)_{j}}{\left(a b c d q^{2 \alpha+k-1}, q^{-\alpha-n} / a b\right)_{j}} \\
\quad \cdot{ }_{8} W_{7}\left(a b c d q^{2 \alpha+k-2} ; a b q^{\alpha-1}, a c q^{\alpha-1}, q^{k+1},\right. \\
\left.a b c d q^{2 \alpha+n+k}, q^{-j} ; q, d q^{j-n-1} / a\right) .
\end{aligned}
$$

This leads to the following expression for $S_{n}$ :

(6.11)

$$
\begin{aligned}
S_{n}= & \frac{\left(c d q^{\alpha}, b d q^{\alpha}, a b c d q^{2 \alpha}, a d q\right)_{n}}{\left(a b q^{\alpha+1}, a c q^{\alpha+1}, a d q^{\alpha+1}, a b c d q^{\alpha}\right)_{n}}(a q / d)^{n} \\
& \cdot \sum_{k=0}^{n} \frac{\left(q^{-n}, a b c d q^{2 \alpha+n}, d z, d / z\right)_{k}}{\left(q, a d q, b d q^{\alpha}, c d q^{\alpha}\right)_{k}} q^{k} \\
& \cdot \sum_{i=0}^{n-k} \frac{\left(a b c d q^{2 \alpha+k-2}, a b q^{\alpha-1}, a c q^{\alpha-1}, q^{k+1}, a b c d q^{2 \alpha+n+k}, q^{k-n}\right)_{i}}{\left(q, c d q^{\alpha+k}, b d q^{\alpha+k}, a b c d q^{2 \alpha-2}, q^{-n}, q^{-n} / a d\right)_{i}} \\
& \cdot \frac{\left(1-a b c d q^{2 \alpha+k-2+2 i}\right)\left(q^{\alpha}, b c q^{\alpha-1}\right)_{i}}{\left(1-a b c d q^{2 \alpha+k-2}\right)\left(a b c d q^{2 \alpha+k-1}\right)_{2 i}}(-1)^{i} q^{\left(\begin{array}{c}
i \\
2
\end{array}\right)}\left(\frac{a q^{n}}{d}\right)^{-i} \\
& \cdot{ }_{4} \phi_{3}\left[\begin{array}{l}
q^{i+k-n}, q^{\alpha+i}, b c q^{\alpha+i-1}, q^{i-n-1} \\
a b c d q^{2 \alpha+2 i+k-1}, q^{i-n}, q^{i-n} / a d
\end{array} ; q, q\right] \cdot
\end{aligned}
$$


Since (6.5),

$$
\begin{aligned}
{ }_{4} \phi_{3}\left[\begin{array}{l}
q^{i+k-n}, q^{\alpha+i}, b c q^{\alpha+i-1}, q^{i-n-1} \\
a b c d q^{2 \alpha+2 i+k+1}, q^{i-n}, q^{i-n} / a d
\end{array} q, q\right] \\
=\frac{\left(q^{\alpha+1}, a d q^{\alpha+1}\right)_{n}\left(q^{-n}, q^{-n} / a d\right)_{i}(q, a d q)_{k}}{(q, q a d ; q)_{n}\left(q^{\alpha+1}, a d q^{\alpha+1}\right)_{i+k}}\left(a d q^{a+n+k+1}\right)^{i} \\
\cdot{ }_{4} \phi_{3}\left[\begin{array}{l}
q^{i+k-n}, a b c d q^{2 \alpha+n+k+i}, a d q^{\alpha+i+k}, q^{\alpha+i} \\
a b c d q^{2 \alpha+2 i+k-1}, q^{\alpha+1+k+i}, a d q^{\alpha+1+k+i}
\end{array} ; q, q\right]
\end{aligned}
$$

we find that

$$
\begin{aligned}
& S_{n}= \frac{\left(b d q^{\alpha}, c d q^{\alpha}, a b c d q^{2 \alpha}, q^{\alpha+1}\right)_{n}}{\left(a b q^{\alpha+1}, a c q^{\alpha+1}, a b c d q^{\alpha}, q\right)_{n}}\left(a q^{1-\alpha} / d\right)^{n} \\
& \cdot \sum_{k=0}^{n} \frac{(d z, d / z)_{k}}{\left(q^{\alpha+1}, a d q^{\alpha+1}, b d q^{\alpha}, c d q^{\alpha}\right)_{k}} q^{(\alpha+1) k} \\
& \cdot \sum_{i=0}^{n-k} \frac{\left(a b c d q^{2 \alpha+k-2}, a b q^{\alpha-1}, a c q^{\alpha-1}, q^{k+1}, b c q^{\alpha-1}, q^{\alpha}\right)_{i}}{\left(q, b d q^{\alpha+k}, c d q^{\alpha+k}, a b c d q^{\alpha+k}, a b c d q^{2 \alpha-2}, q^{\alpha+1+k}, a d q^{\alpha+1+k}\right)_{i}} \\
& \cdot \frac{\left(1-a b c d q^{2 \alpha+k+2 i-2}\right)\left(q^{\alpha+k+1} d^{2}\right)^{i}}{\left(1-a b c d q^{2 \alpha+k-2}\right)\left(a b c d q^{2 \alpha+k-1}\right)_{2 i}} \\
& \cdot(-1)^{i} q^{i(i-1) / 2}\left(q^{-n}, a b c d q^{2 \alpha+n}\right)_{i+k+1}
\end{aligned}
$$

We now substitute the above expression into (6.6), use the definition of $A_{n+\alpha}$ and $C_{n+\alpha}$ from (1.14), and (1.15), respectively, and simplify the coefficients to get

$$
\begin{aligned}
& A_{\alpha+n}\left\{p_{n+1}^{\alpha}(x)-p_{n}^{\alpha}(x)\right\}-C_{\alpha+n}\left\{p_{n}^{\alpha}(x)-p_{n-1}^{\alpha}(x)\right\} \\
&=\left(z+z^{-1}-a-a^{-1}\right) \frac{\left(b d q^{\alpha}, c d q, q^{\alpha+1}\right)_{n}}{\left(a b q^{\alpha}, a c q^{\alpha}, a b c d q^{\alpha-1}\right)_{n}} \\
& \cdot \frac{q^{-\alpha n}(a / d)^{n}}{(a b c d q)_{n}\left(1-a b c d q^{2 \alpha+2 n-1}\right)\left(1-a d q^{\alpha}\right)} \\
& \cdot \sum_{k} \sum_{i} \sum_{j} \cdots\left(q^{1-n}\right)_{i+j+k-1}\left(a b c d q^{2 \alpha-1}\right)_{n+i+j+k} /(q)_{n} \\
& \cdot\left\{\left(1-a d q^{\alpha+n}\right)\left(1-q^{-n}\right)\left(1-a b c d q^{2 \alpha+n+j+k+i-1}\right)\right. \\
&\left.-\left(1-q^{n}\right)\left(1-b c q^{\alpha+n-1}\right)\left(1-q^{j+k+i-n}\right) a d q^{\alpha}\right\}
\end{aligned}
$$

where the summands over $k, j$ and $i$ in (6.14) are the terms on the righthand side of (6.13) that are independent of $n$. The expression within the curly brackets now factors into $\left(1-q^{-n}\right)\left(1-a b c d q^{2 \alpha+2 n-1}\right)\left(1-a d q^{\alpha+j+k+i}\right)$. This converts the right-hand side of $(6.14)$ into the following expression: 
$(6.15)$

$$
\begin{gathered}
-a^{-1}(1-a z)(1-a / z) \frac{\left(b d q^{\alpha}, c d q^{\alpha}, q^{\alpha+1}, a b c d q^{2 \alpha-1}\right)_{n}}{\left(a b q^{\alpha}, a c q^{\alpha}, a b c d q^{\alpha-1}, q\right)_{n}}\left(a / d q^{\alpha}\right)^{n} \\
\cdot \sum_{k=0}^{n} \frac{\left(q^{-n}, a b c d q^{2 \alpha+n-1}, d z, d / z\right)_{k}}{\left(q^{\alpha+1}, a d q^{\alpha}, b d q^{\alpha}, c d q^{\alpha}\right)_{k}} q^{(\alpha+1) k} \\
\cdot \sum_{j=0}^{n-k} \frac{\left(1-a b c d q^{2 \alpha+k+2 j-2}\right)}{\left(1-a b c d q^{2 \alpha+k-2}\right)} \\
\cdot \frac{\left(a b c d q^{2 \alpha+k-2}, a b q^{\alpha-1}, a c q^{\alpha-1}, q^{k+1}, a b c d q^{2 \alpha+n+k-1}, q^{k-n}\right)_{j}}{\left(q, c d q^{\alpha+k}, b d q^{\alpha+k}, a b c d q^{2 \alpha-2}, q^{\alpha+k+1}, a d q^{\alpha+k}\right)_{j}} \\
\cdot \frac{\left(q^{\alpha}, b c q^{\alpha-1}\right)_{j}(-1)^{j} q^{j(j-1) / 2}\left(d^{2} q^{\alpha+k+1}\right)^{j}}{\left(a b c d q^{2 \alpha+k-1}\right)_{2 j}} \\
\cdot{ }_{3} \phi_{2}\left[\begin{array}{c}
q^{j+k-n}, a b c d q^{2 \alpha+n+k+j-1}, q^{\alpha+j} \\
a b c d q^{2 \alpha+2 j+k-1}, q^{\alpha+k+1+j}
\end{array} ;, q\right] .
\end{gathered}
$$

By the $q$-Saalschütz formula [13, (1.7.2)]

$$
\begin{aligned}
{ }_{3} \phi_{2}\left[\begin{array}{c}
q^{i+k-n}, a b c d q^{2 \alpha+n+k+i-1}, q^{\alpha+i} \\
a b c d q^{2 \alpha+2 i+k-1}, q^{\alpha+1+k+i}
\end{array} ; q, q\right] \\
=\frac{\left(a b c d q^{\alpha+i+k-1}, q^{i-n}\right)_{n-i-k}}{\left(a b c d q^{2 \alpha+2 i+k-1}, q^{-\alpha-n}\right)_{n-i-k}} \\
=\frac{q^{\alpha n}\left(a b c d q^{\alpha-1}, q\right)_{n}}{\left(a b c d q^{2 \alpha-1}, q^{\alpha+1}\right)_{n}} \frac{\left.\left(a b c d q^{2 \alpha-1}\right)_{k}\left(q^{\alpha+1} ; q\right)_{i+k}, a b c d q^{2 \alpha+n-1}\right)_{i}(q)_{k}\left(a b c d q^{\alpha-1}\right)_{i+k}}{(-1)^{i} q^{-i(i+1) / 2-\alpha(i+k)-i k}}
\end{aligned}
$$

Substituting (6.16) into (6.15) we find that

$$
\begin{aligned}
A_{n+\alpha}\{ & \left.p_{n+1}^{\alpha}(z)-p_{n}^{\alpha}(z)\right\}-C_{n+\alpha}\left\{p_{n}^{\alpha}(z)-p_{n-1}^{\alpha}(z)\right\} \\
= & \left(z+z^{-1}-a-a^{-1}\right) \frac{\left(b d q^{\alpha}, c d q^{\alpha}\right)_{n}}{\left(a b q^{\alpha}, a c q^{\alpha}\right)_{n}}\left(\frac{a}{d}\right)^{n} \\
& \cdot \sum_{k=0}^{n} \frac{\left(q^{-n}, a b c d q^{2 \alpha+n-1}, a b c d q^{2 \alpha-1}, d z, d / z\right)_{k}}{\left(q, a d q^{\alpha}, b d q^{\alpha}, c d q^{\alpha}, a b c d q^{\alpha-1}\right)_{k}} q^{k}, q^{\alpha}, a b q^{\alpha-1}, a c q^{\alpha-1}, b c q^{\alpha-1}, q^{k+1} \\
& \cdot{ }_{10} W_{9}\left(a b c d q^{2 \alpha+k-2}\right. \\
= & \left(z+z^{-1}-a-a^{-1}\right) \frac{\left(b d q^{\alpha}, c d q^{\alpha}\right)_{n}}{\left(a b q^{\alpha}, a c q^{\alpha}\right)_{n}}\left(\frac{a}{d}\right)^{n} p_{n}^{\alpha}(x ; d, b, c, a \mid q) \\
= & \left(z+z^{-1}-a-a^{-1}\right) p_{n}^{\alpha}(x ; a, b, c, d \mid q)
\end{aligned}
$$

by (6.2). This completes the proof of (4.15). 


\section{AsSOCIATED CONTINUOUS $q$-JACOBI POLYNOMIALS}

Some special cases of the associated Askey-Wilson polynomials are worth noting. One such case is the continuous $q$-Jacobi polynomials when we choose

$$
a=\sqrt{q}, \quad b=q^{\alpha+1 / 2}, \quad c=-q^{\beta+1 / 2}, \quad d=-\sqrt{q}, \quad \alpha=\lambda
$$

in (4.15). Then (4.15) gives

$$
\begin{aligned}
p_{n}^{\lambda}\left(x ; \sqrt{q}, q^{\alpha+1 / 2},-q^{\beta+1 / 2},-\sqrt{q} \mid q\right) \\
=\sum_{k=0}^{n} \frac{\left(q^{-n}, q^{2 \lambda+n+\alpha+\beta+1}, q^{2 \lambda+\alpha+\beta+1}, \sqrt{q} z, \sqrt{q} / z\right)_{k}}{\left(q, q^{\alpha+1+\lambda},-q^{\beta+1+\lambda},-q^{\lambda+1}, q^{\lambda+\alpha+\beta+1}\right)_{k}} q^{k} \\
\quad{ }_{10} W_{9}\left(q^{2 \lambda+\alpha+\beta+k} ; q^{\lambda},-q^{\alpha+\beta+\lambda},-q^{\alpha+\lambda}, q^{\beta+\lambda}, q^{k+1},\right. \\
\left.q^{2 \lambda+\alpha+\beta+1+k+n}, q^{k-n} ; q, q\right) .
\end{aligned}
$$

The continuous $q$-ultraspherical polynomials of $L$. J. Rogers correspond to the case $\alpha=\beta, \lambda=0$. When $\lambda>0, \alpha=\beta$ we get the associated continuous $q$-ultraspherical polynomials [10]. By (6.2) we also have

$$
\begin{gathered}
p_{n}^{\lambda}\left(x ; \sqrt{q}, q^{\alpha+1 / 2},-q^{\beta+1 / 2}, \sqrt{q} \mid q\right)=\frac{\left(q^{\lambda+\beta+1},-q^{\lambda+\alpha+1}\right)_{n}}{\left(q^{\lambda+\alpha+1},-q^{\lambda+\beta+1}\right)_{n}}(-1)^{n} \\
\cdot \sum_{k=0}^{n} \frac{\left(q^{-n}, q^{2 \lambda+n+\alpha+\beta+1}, q^{2 \lambda+\alpha+\beta+1},-\sqrt{q} z,-\sqrt{q} / z\right)_{k}}{\left(q, q^{\beta+1+\lambda},-q^{\alpha+1+\lambda},-q^{\lambda+1}, q^{\lambda+\alpha+\beta+1}\right)_{k}} q^{k} \\
\cdot{ }_{10} W_{9}\left(q^{2 \lambda+\alpha+\beta+k} ; q^{\lambda},-q^{\alpha+\beta+\lambda},-q^{\beta+\lambda}, q^{\alpha+\lambda}, q^{k+1},\right. \\
\left.q^{2 \lambda+\alpha+\beta+1+k+n}, q^{k-n} ; q, q\right) .
\end{gathered}
$$

In both cases the ${ }_{10} W_{9}$ series are terminating and balanced, and hence can be transformed by Bailey's formula, Gasper and Rahman [13, (2.9.1)]. Thus

$$
\begin{aligned}
&{ }_{10} W_{9}\left(q^{2 \lambda+\alpha+\beta+k} ; q^{k+1}, q^{\alpha+\lambda},-q^{\alpha+\beta+\lambda},-q^{\beta+\lambda}, q^{\lambda},\right.\left.q^{2 \lambda+\alpha+\beta+1+n+k}, q^{k-n} ; q, q\right) \\
&= \frac{\left(q^{2 \lambda+\alpha+\beta+1+k},-q^{\alpha+1},-q^{\beta+\lambda+1}, q^{\lambda+1}\right)_{n}}{\left(-q^{\alpha+\lambda+1}, q^{\lambda+\alpha+\beta+1}, q,-q^{2 \lambda+\beta+1}\right)_{n}} \\
& \cdot \frac{\left(q^{\lambda+\alpha+\beta+1},-q^{\lambda+\alpha+1},-q^{2 \lambda+\beta+1}, q\right)_{k}}{\left(q^{2 \lambda+\alpha+\beta+1},-q^{\alpha+1},-q^{\lambda+\beta+1}, q^{\lambda+1}\right)_{k}} \\
& \cdot{ }_{10} W_{9}\left(-q^{2 \lambda+\beta+k} ;-q^{k+1-\alpha},-q^{\lambda}, q^{\beta+\lambda},-q^{\beta+\lambda}, q^{\lambda},\right. \\
&\left.q^{2 \lambda+\alpha+\beta+1+n+k}, q^{k-n} ; q, q\right) .
\end{aligned}
$$


Hence it follows from (7.3) and (7.4) that

$$
\begin{aligned}
p_{n}^{\lambda}(x ; & \left.\sqrt{q}, q^{\alpha+1 / 2},-q^{\beta+1 / 2},-\sqrt{q} \mid q\right) \\
= & \frac{\left(q^{2 \lambda+\alpha+\beta+1},-q^{\alpha+1}, q^{\lambda+\beta+1}, q^{\lambda+1}\right)_{n}}{\left(q^{\alpha+\lambda+1}, q^{\lambda+\alpha+\beta+1}, q,-q^{2 \lambda+\beta+1}\right)_{n}} \\
& \cdot \sum_{k=0}^{n} \frac{\left(q^{-n}, q^{2 \lambda+\alpha+\beta+1+n},-q^{2 \lambda+\beta+1},-\sqrt{q} z,-\sqrt{q} / z\right)_{k}}{\left(q^{\beta+\lambda+1},-q^{\beta+\lambda+1},-q^{\alpha+1}, q^{\lambda+1},-q^{\lambda+1}\right)_{k}} q^{k},-q^{k+1-\alpha},-q^{\lambda}, q^{\beta+\lambda},-q^{\beta+\lambda}, q^{\lambda}, \\
& \left.\cdot{ }_{10}^{2 \lambda+\alpha+\beta+1+n+k}, q^{k-n} ; q, q\right) .
\end{aligned}
$$

The explicit representation (7.5) is a $q$-analogue of a formula Wimp [33] obtained for associated Jacobi polynomials.

\section{EXPLICIT FORM OF $q_{n}^{\alpha}(x ; q, b, c, d \mid q)$}

It is obvious that $p_{n}^{\alpha}(x)$ and $q_{n}^{\alpha}(x)$ both satisfy the same recurrence relation, namely

$$
\begin{aligned}
\left(z+z^{-1}-a-a^{-1}+A_{\alpha+n}+C_{\alpha+n}\right) y_{n}^{\alpha}(x) \\
\quad=A_{\alpha+n} y_{n+1}^{\alpha}(x)+C_{\alpha+n} y_{n-1}^{\alpha}(x), \quad \text { for } n=0,1, \ldots
\end{aligned}
$$

They, of course, satisfy two different initial conditions, namely

$$
p_{0}^{\alpha}(x)=1, \quad p_{-1}^{\alpha}(x)=0
$$

and

$$
q_{0}^{\alpha}(x)=1, \quad q_{1}^{\alpha}(x)=1+A_{\alpha}^{-1}\left(z+z^{-1}-a-a^{-1}\right)
$$

We shall prove that

$$
q_{n}^{\alpha}(x)=p_{n}^{\alpha}(x)-\frac{C_{\alpha}}{A_{\alpha}} p_{n-1}^{\alpha+1}(x)
$$

It is clear that $q_{1}^{\alpha}(x)=p_{1}^{\alpha}(x)-C_{\alpha} / A_{\alpha}$, so (8.2) holds for $n=0,1$. Since $p_{n}^{\alpha}(x)$ and $p_{n-1}^{\alpha+1}(x)$ satisfy $(8.1)$ it follows that the right-hand side of $(8.2)$ is a solution of $(8.1)$ which agrees with $q_{n}^{\alpha}(x)$ initially. Thus (8.2) holds. 
Hence, by (4.15)

(8.3)

$$
\begin{aligned}
& q_{n}^{\alpha}(x ;a, b, c, d \mid q) \\
&= \sum_{k=0}^{n} \frac{\left(q^{-n}, a b c d q^{2 \alpha+n-1}, a b c d q^{2 \alpha-1}, a z, a / z\right)_{k}}{\left(q, a b q^{\alpha}, a c q^{\alpha}, a d q^{\alpha}, a b c d q^{\alpha-1}\right)_{k}} q^{k} \\
& \cdot{ }_{10} W_{9}\left(a b c d q^{2 \alpha+k-2} ; q^{\alpha}, b c q^{\alpha-1}, b d q^{\alpha-1}, c d q^{\alpha-1}, q^{k+1},\right. \\
&- \frac{\left(1-b c q^{\alpha-1}\right)\left(1-b d q^{\alpha-1}\right)\left(1-c d q^{\alpha-1}\right)\left(1-q^{\alpha}\right)\left(1-a b c d q^{2 \alpha}\right)}{\left(1-a b q^{\alpha}\right)\left(1-a c q^{\alpha}\right)\left(1-a d q^{\alpha}\right)\left(1-a b c d q^{\alpha-1}\right)\left(1-a b c d q^{2 \alpha-2}\right)} a^{2} \\
&\left.\cdot \sum_{k=0}^{n-1} \frac{\left(q^{1-n}, a b c d q^{2 \alpha+n}, a b c d q^{2 \alpha+1}, a z, a / z\right)_{k}}{\left(q, a b q^{\alpha+1}, a c q^{\alpha+1}, a d q^{\alpha+1}, a b c d q^{\alpha}\right)_{k}} q^{2+n}, q^{k-n} ; q, a^{2}\right) \\
& \cdot{ }_{10} W_{9}\left(a b c d q^{2 \alpha+k} ; q^{\alpha+1}, b c q^{\alpha}, b d q^{\alpha}, c d q^{\alpha}, q^{k+1},\right. \\
&\left.a b c d q^{2 \alpha+n+k}, q^{k-n+1} ; q, a^{2}\right)
\end{aligned}
$$

for $n=1,2, \ldots$. We shall now combine the two double series on the righthand side of (8.3) and express $q_{n}^{\alpha}(x)$ as a linear combination of ${ }_{10} W_{9}$ 's. First, let us rewrite $(8.3)$ in the expanded form

$$
\begin{aligned}
q_{n}^{\alpha}(x)= & \sum_{k=0}^{n} \sum_{j=0}^{n-k} \frac{\left(a b c d q^{2 \alpha-1}, a z, a / z\right)_{k} q^{k}}{(q)_{k}} \\
& \cdot \frac{\left(1-a b c d q^{2 \alpha+k-2+2 j}\right)\left(a b c d q^{2 \alpha+k-2}, q^{\alpha}, b c q^{\alpha-1}, b d q^{\alpha-1}, c d q^{\alpha-1}\right)_{j}}{\left(1-a b c d q^{2 \alpha+k-2}\right)(q)_{j}\left(a b c d q^{\alpha-1}, a b q^{\alpha}, a c q^{\alpha}, a d q^{\alpha}\right)_{j+k}} \\
& \cdot \frac{\left(q^{k+1}\right)_{j}\left(q^{-n}, a b c d q^{2 \alpha+n-1}\right)_{j+k} a^{2 j}}{\left(a b c d q^{2 \alpha-2}\right)_{j}\left(q^{-n}, a b c d q^{2 \alpha+n-1}\right)_{j}} \\
- & \sum_{k=0}^{n-1} \sum_{j=0}^{n-1-k} \frac{\left(a b c d q^{2 \alpha+1}, a z, a / z\right)_{k} q^{k}}{(q)_{k}} \\
& \cdot \frac{\left(1-a b c d q^{2 \alpha}\right)\left(1-a b c d q^{2 \alpha+k+2 j}\right)\left(a b c d q^{2 \alpha+k}, q^{k+1}\right)_{j}\left(q^{\alpha}, b c q^{\alpha-1}\right)_{j+1}}{\left(1-a b c d q^{2 \alpha-2}\right)\left(1-a b c d q^{2 \alpha+k}\right)\left(q, a b c d q^{2 \alpha}\right)_{j}\left(a b q^{\alpha}, a c q^{\alpha}\right)_{j+k+1}} \\
& \cdot \frac{\left(b d q^{\alpha-1}, c d q^{\alpha-1}\right)_{j+1}\left(q^{1-n}, a b c d q^{2 \alpha+n}\right)_{j+k}}{\left(a d q^{\alpha}, a b c d q^{\alpha-1}\right)_{j+k+1}\left(q^{1-n}, a b c d q^{2 \alpha+n}\right)_{j}} a^{2(j+1)} \cdot
\end{aligned}
$$

The key step now is to separate the $j=0$ term from the first term on the right-hand side and combine the rest of the term with the second term. Thus 
we find that

$$
\begin{aligned}
q_{n}^{\alpha}(x)= & \sum_{k=0}^{n} \frac{\left(q^{-n}, a b c d q^{2 \alpha+n-1}, a b c d q^{2 \alpha-1}, a z, a / z\right)_{k}}{\left(q, a b q^{\alpha}, a c q^{\alpha}, a d q^{\alpha}, a b c d q^{\alpha-1}\right)_{k}} q^{k} \\
+ & \sum_{k=0}^{n-1} \sum_{j=0}^{n-1-k} \frac{(a z, a / z)_{k} q^{k}\left(q^{\alpha}, b c q^{\alpha-1}, b d q^{\alpha-1}, c d q^{\alpha-1}\right)_{j+1}}{(q)_{k}\left(a b c d q^{\alpha-1}, a b q^{\alpha}, a c q^{\alpha}, a d q^{\alpha}\right)_{j+k+1}} \\
& \cdot \frac{\left(q^{1-n}, a b c d q^{2 \alpha+n}\right)_{j+k}\left(1-a b c d q^{2 \alpha+k+2 j}\right)}{\left(q^{1-n}, a b c d q^{2 \alpha+n}\right)_{j}} a^{2 j+2} E_{j, k},
\end{aligned}
$$

where

$$
\begin{aligned}
E_{j, k}= & \frac{\left(a b c d q^{2 \alpha-1}\right)_{k}\left(a b c d q^{2 \alpha+k-2}, q^{k+1}\right)_{j+1}}{\left(1-a b c d q^{2 \alpha-2+k}\right)\left(q, a b c d q^{2 \alpha-2}\right)_{j+1}} \\
& -\frac{\left(a b c d q^{2 \alpha+1}\right)_{k}\left(1-a b c d q^{2 \alpha}\right)\left(a b c d q^{2 \alpha+k}, q^{k+1}\right)_{j}}{\left(1-a b c d q^{2 \alpha-2}\right)\left(1-a b c d q^{2 \alpha+k}\right)\left(q, a b c d q^{2 \alpha}\right)_{j}} \\
= & \frac{\left(a b c d q^{2 \alpha-1}\right)_{j+k}\left(q^{k+1}\right)_{j+1}}{\left(q, a b c d q^{2 \alpha-2}\right)_{j+1}}-\frac{\left(a b c d q^{2 \alpha}\right)_{j+k}\left(q^{k+1}\right)_{j}}{\left(1-a b c d q^{2 \alpha-2}\right)\left(q, a b c d q^{2 \alpha}\right)_{j}}
\end{aligned}
$$

Using the factorization

$$
\begin{aligned}
& \left(1-q^{j+k+1}\right)\left(1-a b c d q^{2 \alpha+j-1}\right)-\left(1-q^{j+1}\right)\left(1-a b c d q^{2 \alpha+j+k-1}\right) \\
& =q^{j+1}\left(1-q^{k}\right)\left(1-a b c d q^{2 \alpha-2}\right)
\end{aligned}
$$

it follows that

$$
E_{j, k}=\frac{\left(a b c d q^{2 \alpha-1}\right)_{j+k}\left(q^{k}\right)_{j+1}}{\left(q, a b c d q^{2 \alpha-1}\right)_{j+1}} q^{j+1}
$$

Substituting (8.7) into (8.5) we obtain

$$
\begin{aligned}
q_{n}^{\alpha}(x) & =\sum_{k=0}^{n} \frac{\left(q^{-n}, a b c d q^{2 \alpha+n-1}, a b c d q^{2 \alpha-1}, a z, a / z\right)_{k}}{\left(q, a b q^{\alpha}, a c q^{\alpha}, a d q^{\alpha}, a b c d q^{\alpha-1}\right)_{k}} q^{k} \\
& +\sum_{k=0}^{n-1} \sum_{j=0}^{n-1-k} \frac{(a z, a / z)_{k} q^{k}\left(a b c d q^{2 \alpha-2}, q^{-n}, a b c d q^{2 \alpha+n-1}\right)_{j+k+1}}{(q)_{k}\left(a b c d q^{\alpha-1}, a b q^{\alpha}, a c q^{\alpha}, a d q^{\alpha}\right)_{j+k+1}} \\
& \cdot \frac{\left(q^{\alpha}, b c q^{\alpha-1}, b d q^{\alpha-1}, c d q^{\alpha-1}, q^{k}\right)_{j+1}\left(1-a b c d q^{2 \alpha+k+2 j}\right)}{\left(q^{-n}, a b c d q^{2 \alpha+n-1}, a b c d q^{2 \alpha-1}, q\right)_{j+1}\left(1-a b c d q^{2 \alpha-2}\right)}\left(q a^{2}\right)^{j+1}
\end{aligned}
$$


It is obvious that the single series on the right-hand side is the one that one would obtain by setting $j+1=0$ in the second series. So, after replacing $j+1$ by $j$ in the second series we may combine the two terms to obtain the following expression

$$
\begin{aligned}
& q_{n}^{\alpha}(x ;a, b, c, d \mid q) \\
&= \sum_{k=0}^{n} \frac{\left(q^{-n}, a b c d q^{2 \alpha+n-1}, a b c d q^{2 \alpha-1}, a z, a / z\right)_{k}}{\left(q, a b q^{\alpha}, a c q^{\alpha}, a d q^{\alpha}, a b c d q^{\alpha-1}\right)_{k}} q^{k} \\
& \cdot{ }_{10} W_{9}\left(a b c d q^{2 \alpha-2+k} ; q^{\alpha}, b c q^{\alpha-1}, b d q^{\alpha-1}, c d q^{\alpha-1},\right. \\
&\left.q^{k}, a b c d q^{2 \alpha+n+k-1}, q^{k-n} ; q, q a^{2}\right) .
\end{aligned}
$$

When $a=q^{1 / 2}$, the ${ }_{10} W_{9}$ series on the right-hand side of (8.9) is not balanced and hence cannot be transformed directly into another ${ }_{10} W_{9}$ series as we did in (7.4). However, we could use the representation (8.3) where both ${ }_{10} W_{9}$ series are balanced when $a=q^{1 / 2}$ and hence can be transformed in the same way as in (7.4). It can then be shown by the same procedure as used in the previous section that as $q \rightarrow 1$,

$$
q_{n}^{\lambda}\left(x ; q^{1 / 2}, q^{\alpha+1 / 2},-q^{\beta+1 / 2},-q^{1 / 2} \mid q\right)
$$

approaches the formula (3.4) in Ismail and Masson [16].

\section{Miscellaneous Results}

We mentioned before that for $|z|<1$, the ${ }_{8} W_{7}$ series in the expression (4.13) for $p_{n}^{\alpha}(x ; a, b, c, d \mid q)$ are convergent provided $0<q<|a|<1$. This restriction can be weakened if we use the transformation

$$
\begin{aligned}
&{ }_{8} W_{7}\left(b c d / z q ; b / z, c / z, d / z, a b c d q^{\alpha+n-1}, q^{-\alpha-n} ; q, q z / a\right) \\
&= \frac{(b c d / z, b z, c q / a, d q / a)_{\infty}}{(b c, b d, q z / a, c d q / a z)_{\infty}} \\
& \cdot{ }_{8} W_{7}\left(c d / a z ; c / z, d / z, q / a z, c d q^{\alpha+n}, q^{1-\alpha-n} / a b ; q, b z\right),
\end{aligned}
$$

which follows by applying (4.30). Since the left-hand side is symmetric in $b, c, d$ the right-hand side must have the same property, which means that there are two other forms of the expression on the right-hand side with $b, c, d$ interchanged. The use of (9.1) gives the following alternative form of (4.13) 


$$
\begin{aligned}
& p_{n}^{\alpha}(x ; a,b, c, d \mid q) \\
&= \frac{\left(c d, c q / a, d q / a, b c q^{\alpha-1}, b d q^{\alpha-1}, c d q^{\alpha-1}, q^{\alpha}, a z\right)_{\infty}}{\left(a b q^{\alpha}, a c q^{\alpha}, a d q^{\alpha}, a b c d q^{\alpha-1}, q z / a, c z, d z, c d q / a z\right)_{\infty}} \frac{z a^{n}}{\left(1-a b c d q^{2 \alpha-2}\right)} \\
& \cdot\left\{\frac{\left(b c d q^{\alpha+n} / z\right)_{\infty}}{\left(a z q^{\alpha+n}\right)_{\infty}} z^{-n-1}\right. \quad \frac{\left(a b c d q^{2 \alpha-2}, b z q^{\alpha}, c z q^{\alpha}, d z q^{\alpha}, b c d z q^{\alpha-1}, a b q^{\alpha+n}, a c q^{\alpha+n}, a d q^{\alpha+n}\right)_{\infty}}{\left(b c q^{\alpha-1}, b d q^{\alpha-1}, c d q^{\alpha-1}, q^{\alpha}, b c d z q^{2 \alpha-1}, b c q^{\alpha+n}, b d q^{\alpha+n}, c d q^{\alpha+n}\right)_{\infty}} \\
& \cdot{ }_{8} W_{7}\left(b c d z q^{2 \alpha-2} ; b c q^{\alpha-1}, b d q^{\alpha-1}, c d q^{\alpha-1}, q^{\alpha}, z q / a ; q, a z\right) \\
& \cdot{ }_{8} W_{7}\left(c d / a z ; c / z, d / z, q / a z, c d q^{\alpha+n}, q^{1-\alpha-n} / a b ; q, b z\right) \\
& \quad \frac{\left(a b c d q^{2 \alpha+2 n}, b z q^{\alpha+n+1}, c z q^{\alpha+n+1}, d z q^{\alpha+n+1}, b c d z q^{\alpha+n}, a b q^{\alpha-1}\right)_{\infty}}{\left(b c q^{\alpha+1}, b d q^{\alpha+n}, c d q^{\alpha+n}, q^{\alpha+n+1}, b c d z q^{2 \alpha+2 n+1}, b c q^{\alpha-1}, b d q^{\alpha-1}\right)_{\infty}} \\
& \cdot \frac{\left(a c q^{\alpha-1}, a d q^{\alpha-1}, b c d q^{\alpha-1} / z\right)_{\infty}}{\left(c d q^{\alpha-1}, a z q^{\alpha-1}\right)_{\infty}} z^{n+1} \\
& \cdot{ }_{8} W_{7}\left(b c d z q^{2 \alpha+2 n} ; b c q^{\alpha+n}, b d q^{\alpha+n}, c d q^{\alpha+n}, q^{\alpha+n+1}, z q / a ; q, a z\right) \\
&\left.\cdot{ }_{8} W_{7}\left(c d / a z ; c / z, d / z, q / a z, c d q^{\alpha-1}, q^{2-\alpha} / a b ; q, b z\right)\right\}
\end{aligned}
$$

Note that the ${ }_{8} W_{7}$ series in (9.2) are convergent when $|z|<1$ and $\max (|a|,|b|)$ $<1$, and hence (9.2) constitutes an analytic continuation of (4.13).

Now we will show how to obtain a complete asymptotic expansion of $p_{n}^{\alpha}(x ; a, b, c, d \mid q)$ from (4.13), when $|z|<1$. The important step is to use Bailey's formula $\left[9,8.5(3)\right.$, p. 69] that expresses a very well-poised ${ }_{8} \phi_{7}$ series in terms of two balanced nonterminating ${ }_{4} \phi_{3}$ series. Thus, we have

$$
\begin{aligned}
& { }_{8} W_{7}\left(b c d / z q ; b / z, c / z, d / z, a b c d q^{\alpha+n-1}, q^{-\alpha-n} ; q, q z / a\right) \\
& =\frac{(b c d / z, b z, c z, d z)_{\infty}}{\left(b c, b d, c d, z^{2}\right)_{\infty}} \phi_{3}\left[\begin{array}{c}
b / z, c / z, d / z, q / a z \\
q / z^{2}, b c d q^{\alpha+n} / z, q^{-1-\alpha-n} / a z
\end{array} ; q, q\right] \\
& +\frac{\left(b c d / z, b / z, c / z, d / z, q / a z, b c d z q^{\alpha+n}, z q^{1-\alpha-n} / a\right)_{\infty}}{\left(c d, b d, b c, b c d q^{\alpha+n} / z, q^{1-\alpha-n} / a z, q z / a, z^{-2}\right)_{\infty}} \\
& \cdot{ }_{4} \phi_{3}\left[\begin{array}{c}
b z, c z, d z, q z / a \\
q z^{2}, b c d z q^{\alpha+n}, z q^{1-\alpha-n} / a
\end{array} ; q, q\right]
\end{aligned}
$$

and

$$
\begin{aligned}
&{ }_{8} W_{7}\left(b c d z q^{2 \alpha+2 n} ; b c q^{\alpha+n}, b d q^{\alpha+n}, c d q^{\alpha+n}, q^{\alpha+n+1}, z q / a ; q, a z\right) \\
&= \frac{\left(b c d z q^{2 \alpha+2 n+1}, a b c d q^{\alpha+n-1}, a z q^{\alpha+n+1}, q z^{2}\right)_{\infty}}{\left(b c d z q^{\alpha+n}, a b c d q^{2 \alpha+2 n}, a z, z^{2} q^{\alpha+n+2}\right)_{\infty}} \\
& \cdot{ }_{8} W_{7}\left(z^{2} q^{\alpha+n+1} ; z q / a, z q / b, z q / c, z q / d, q^{\alpha+n+1} ; q, a b c d q^{\alpha+n-1}\right),
\end{aligned}
$$


by (4.30). Thus the left-hand side of (9.4) is given by

$$
\begin{aligned}
& \text { L.H.S. of }(9.4)=\frac{\left(b c q^{\alpha+n}, b d q^{\alpha+n}, c d q^{\alpha+n}\right)_{\infty}}{\left(b z q^{\alpha+n+1}, c z q^{\alpha+n+1}, d z q^{\alpha+n+1}\right)_{\infty}} \\
& \cdot \frac{\left(b c d z q^{2 \alpha+2 n+1}, a b c d q^{\alpha+n-1}, a z q^{\alpha+n+1}, q z^{2}\right)_{\infty}}{\left(b c d z q^{\alpha+n}, a b c d q^{2 \alpha+2 n}, a z, b c d q^{\alpha+n-1} / z\right)_{\infty}} \\
& \cdot{ }_{4} \phi_{3}\left[\begin{array}{c}
z q / b, z q / c, z q / d, a z \\
q z^{2}, a z q^{\alpha+n+1}, z q^{2-\alpha-n} / b c d
\end{array} ; q, q\right] \\
& +\frac{\left(z q / b, z q / c, z q / d, b c d z q^{2 \alpha+2 n+1}\right)_{\infty}}{\left(b z q^{\alpha+n+1}, c z q^{\alpha+n+1}, d z q^{\alpha+n+1}, z q^{1-\alpha-n} / b c d\right)_{\infty}}
\end{aligned}
$$

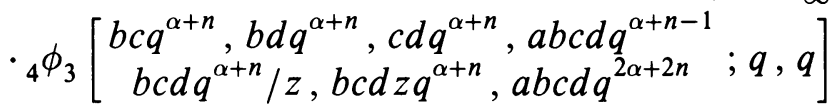

Using (9.3), (9.4), and (9.5) in (4.13) and simplifying the coefficients we obtain the complete asymptotic expansion

$$
\begin{aligned}
& p_{n}^{\alpha}(x ; a, b, c, d \mid q) \\
& =\frac{\left(a b c d q^{2 \alpha-1}, b c, b d, c d, a z, b z q^{\alpha}, c z q^{\alpha}, d z q^{\alpha}, b c d z q^{\alpha-1}, b c d q^{\alpha} / z\right)_{\infty}}{\left(a b c d q^{\alpha-1}, b c q^{\alpha}, b d q^{\alpha}, c d q^{\alpha}, a z q^{\alpha}, b c d z q^{2 \alpha-1}, b z, c z, d z, b c d / z\right)_{\infty}} \\
& \frac{\left(b c q^{\alpha}, b d q^{\alpha}, c d q^{\alpha}, a z q^{\alpha}\right)_{n}}{\left(a b q^{\alpha}, a c q^{\alpha}, a d q^{\alpha}, b c d q^{\alpha} / z\right)_{n}}(a / z)^{n} \\
& { }_{8} W_{7}\left(b c d z q^{2 \alpha-2} ; b c q^{\alpha-1}, b d q^{\alpha-1}, c d q^{\alpha-1}, q^{\alpha}, z q / a ; q, a z\right) \\
& \text {. }\left\{\frac{(b z, c z, d z, b c d / z)_{\infty}}{\left(b c, b d, c d, z^{2}\right)_{\infty}} \phi_{3}\left[\begin{array}{c}
b / z, c / z, d / z, q / a z \\
q / z^{2}, b c d q^{\alpha+n}, q^{1-\alpha-n} / a z
\end{array} ; q, q\right]\right. \\
& +\frac{\left(b / z, c / z, d / z, b c d / z, q / a z, b c d z q^{\alpha}, z q^{1-\alpha} / a\right)_{\infty}}{\left(b c, b d, c d, 1 / z^{2}, b c d q^{\alpha} / z, q^{1-\alpha} / a z, q z / a\right)_{\infty}} \\
& \left.\cdot \frac{\left(b c d q^{\alpha} / z, a q^{a} / z\right)_{n}}{\left(a z q^{\alpha}, b c d z q^{\alpha}\right)_{n}} z^{2 n}{ }_{4} \phi_{3}\left[\begin{array}{c}
b z, c z, d z, q z / a \\
q z^{2}, b c d z q^{\alpha+n}, z q^{1-\alpha-n} / a
\end{array} ; q, q\right]\right\} \\
& -\frac{\left(1-a b q^{\alpha-1}\right)\left(1-a c q^{\alpha-1}\right)\left(1-a d q^{\alpha-1}\right)}{1-a b c d q^{2 \alpha-2}} \\
& \cdot \frac{\left(b c, b d, c d, q^{\alpha}, a b c d q^{2 \alpha}, a z\right)_{\infty}}{\left(a b c d q^{\alpha-1}, b c q^{\alpha}, b d q^{\alpha}, c d q^{\alpha}, q^{\alpha+1}, a z q^{\alpha+1}\right)_{\infty}} \\
& \cdot \frac{\left(b z q^{\alpha+1}, c z q^{\alpha+1}, d z q^{\alpha+1}, b c d z q^{\alpha}, b c d q^{\alpha-1} / z\right)_{\infty}}{\left(b z, c z, d z, b c d / z, b c d z q^{2 \alpha+1}\right)_{\infty}} \\
& \cdot \frac{\left(b c q^{\alpha}, b d q^{\alpha}, c d q^{\alpha}, q^{\alpha+1}\right)_{n}\left(b c d z q^{2 \alpha+1}\right)_{2 n}}{\left(b z q^{\alpha+1}, c z q^{\alpha+1}, d z q^{\alpha+1}, b c d z q^{\alpha}\right)_{n}\left(a b c d q^{2 \alpha}\right)_{2 n}} a^{n} z^{n+2}
\end{aligned}
$$




$$
\begin{aligned}
& { }_{8} W_{7}\left(b c d / z q ; b / z, c / z, d / z, a b c d q^{\alpha-2}, q^{1-\alpha} ; q, q z / a\right) \\
& \text { - }\left\{\frac{\left(b c q^{\alpha}, b d q^{\alpha}, c d q^{\alpha}, a b c d q^{\alpha-1}, a z q^{\alpha+1}, b c d z q^{2 \alpha+1}, q z^{2}\right)_{\infty}}{\left(a b c d q^{2 \alpha}, a z, b z q^{\alpha+1}, c z q^{\alpha+1}, d z q^{\alpha+1}, b c d z q^{\alpha}, b c d q^{\alpha} / z\right)_{\infty}}\right. \\
& \frac{\left(b z q^{\alpha+1}, c z q^{\alpha+1}, d z q^{\alpha+1}, b c d z q^{\alpha}, b c d q^{\alpha-1}\right)_{n}\left(a b c d q^{2 \alpha}\right)_{2 n}}{\left(b c q^{\alpha}, b d q^{\alpha}, c d q^{\alpha}, a b c d q^{\alpha-1}, a z q^{\alpha+1}\right)_{n}\left(b c d z q^{2 \alpha+1}\right)_{2 n}} \\
& \cdot{ }_{4} \phi_{3}\left[\begin{array}{c}
z q / b, z q / c, z q / d, a z \\
q z^{2}, a z q^{\alpha+n+1}, z q^{2-\alpha-n} / b c d
\end{array} ; q, q\right] \\
& +\frac{\left(z q / b, z q / c, z q / d, b c d z q^{2 \alpha+1}\right)_{\infty}}{\left(b z q^{\alpha+1}, c z q^{\alpha+1}, d z q^{\alpha+1}, z q^{1-\alpha} / b c d\right)_{\infty}} \\
& \cdot \frac{\left(b z q^{\alpha+1}, c z q^{\alpha+1}, d z q^{\alpha+1}\right)_{n}}{\left(b c d q^{\alpha} / z\right)_{n}\left(b c d z q^{2 \alpha+1}\right)_{2 n}}(-1)^{n} q^{n(n+1) / 2+n \alpha} z^{-n} \\
& \left.{ }_{4} \phi_{3}\left[\begin{array}{c}
b c q^{\alpha+n}, b d q^{\alpha+n}, c d q^{\alpha+n}, a b c d q^{\alpha+n-1} \\
a b c d q^{2 \alpha+2 n}, b c d z q^{\alpha+n}, b c d q^{\alpha+n} / z
\end{array} ; q, q\right]\right\} .
\end{aligned}
$$

We conclude this section by proving a theorem concerning the positivity of linearization coefficients for a special family of associated Askey-Wilson polynomials.

Theorem 4. If $a=-b, c=-d$ then the linearization coefficients

$$
c_{m, n, k}^{\alpha}(-b, b,-d, d)
$$

in (1.29) are nonnegative when $-q<b, d<q$ and $\alpha \geq 0$.

Proof. We apply Askey's theorem, Theorem 2. In the present cases, $\alpha_{n}$ and $\beta_{n}$ of (1.30) are $\alpha_{n}=0, \beta_{n}=A_{n+\alpha-1} C_{n+\alpha}$, with $A_{\alpha}, C_{\alpha}$ as in (1.14) and (1.15). We have

$$
\beta_{n}=\frac{(1-y)\left(q+b^{2} y\right)\left(q+d^{2} y\right)\left(q^{2}-b^{2} d^{2} y\right)}{\left(q-b^{2} d^{2} y^{2}\right)\left(q^{3}-b^{2} d^{2} y^{2}\right)}, \quad y:=q^{n+\alpha} .
$$

To show that $\beta_{n}$ increases with $n$ we show that $\beta_{n}$ decreases with $y$ for $0<$ $y<1$. Let $D$ and $N$ be the denominator and numerator of $\beta_{n}$, respectively. MACSYMA was used to prove that

$$
D \frac{d N}{d y}-N \frac{d D}{d y}=A y^{6}+B y^{5}+C y^{4}+D y^{2}+E y+F
$$

with

$$
\begin{aligned}
& A=b^{6} d^{6}\left(q-b^{2}\right)\left(q-d^{2}\right), \quad F=-q^{6}\left(q-b^{2}\right)\left(q-d^{2}\right), \\
& B=2 q b^{4} d^{4} H, \quad E=-2 q^{5} G, \\
& G=-b^{2} d^{2} q^{2}+d^{2} q^{2}+b^{2} q^{2}-2 b^{2} d^{2} q+b^{2} d^{4}+d^{2} b^{4}-b^{2} d^{2}, \\
& C=q b^{4} d^{4} H, \quad D=-q^{3} b^{2} d^{2} H,
\end{aligned}
$$




$$
\begin{gathered}
H=q^{4}-q^{3}\left(b^{2}+d^{2}\right)+3 q^{3}+q^{2} b^{2} d^{2}-3 q^{2}\left(b^{2}+d^{2}\right) \\
+q^{2}+3 q b^{2} d^{2}-q\left(b^{2}+d^{2}\right)+b^{2} d^{2}
\end{gathered}
$$

Simple manipulations lead to

$$
\begin{aligned}
& G=b^{2}\left(1-d^{2}\right)\left(q^{2}-d^{2}\right)+d^{2}\left(1-b^{2}\right)\left(q^{2}-b^{2}\right)+b^{2} d^{2}(1-q)^{2}, \\
& H=\left(q-b^{2}\right)\left(q-d^{2}\right)\left(1+3 q+q^{2}\right),
\end{aligned}
$$

and we find that $\frac{d \beta_{n}}{d y}$ is a negative multiple of the positive quantity

$\left(q-b^{2}\right)\left(q-d^{2}\right)\left(q^{6}-b^{6} d^{6} y^{6}\right)+y^{2} q b^{2} d^{2} H\left(q^{2}-y^{2} b^{2} d^{2}\right)+2 y q G\left(q^{4}-b^{4} d^{4} y^{4}\right)$,

since $0<y<1$. This shows that $\beta_{n}$ increases with $n$ and Theorem 4 follows from Theorem 2 .

Remark. We believe that the coefficients $c_{m, n, k}^{\alpha}(-b, b,-d, d)$ in (1.29) are nonnegative in the region $-q^{1 / 2}<b, d<q^{1 / 2}$ and $\alpha \geq 0$ but the method of proof of Theorem 4 does not seem to be sharp enough to prove this stronger result.

\section{ACKNOWLEDGMENT}

We thank Richard Askey and Jairo Charris for their interest in this work and their helpful comments. We are grateful to the referee for his suggestions and remarks. We wish to thank Rose Dorch and Frieda Keaton for her excellent typing and patience. Thanks to John Pederson for his help with Macsyma.

\section{REFERENCES}

1. G. Andrews and R. Askey, Classical orthogonal polynomials, Polynômes Orthogonaux et Applications (C. Brezinski et al., eds.), Lecture Notes in Math., vol. 1171, Springer-Verlag, Berlin and New York, 1985, pp. 36-82.

2. R. Askey, Linearization of the product of orthogonal polynomials, Problems in Analysis (R. Gunning, ed.), Princeton Univ. Press, Princeton, N.J., 1970, pp. 223-228.

3. __ Orthogonal polynomials and special functions, Regional Conf. Ser. Appl. Math., SIAM, Philadelphia, Pa., 1975.

4. R. Askey and M. E. H. Ismail, Permutation problems and special functions, Canad. J. Math. 28 (1976), 853-874.

5. __ Recurrence relations, continued fractions and orthogonal polynomials, Mem. Amer. Math. Soc., no. 300, 1984.

6. R. Askey and J. A. Wilson, A set of orthogonal polynomials that generalize the Racah coefficients or 6-j symbols, SIAM J. Math. Anal. 10 (1979), 1008-1016.

7. __ Some basic hypergeometric orthogonal polynomials that generalize Jacobi polynomials, Mem. Amer. Math. Soc., no. 319, 1985.

8. R. Askey and J. Wimp, Associated Laguerre and Hermite polynomials, Proc. Roy. Soc. Edinburgh Sect. A 96 (1984), 15-37.

9. W. N. Bailey, Generalized hypergeometric series, Cambridge Univ. Press, reprinted by Hafner, New York, 1972. 
10. J. Bustoz and M. E. H. Ismail, The associated ultraspherical orthogonal polynomials and their q-analogues, Canad. J. Math. 34 (1982), 718-736.

11. T. Chihara, On co-recursive orthogonal polynomials, Proc. Amer. Math. Soc. 8 (1957), 899905.

12. __ An introduction to orthogonal polynomials, Gordon and Breach, New York, 1978.

13. G. Gasper and M. Rahman, Basic hypergeometric series, Cambridge Univ. Press, Cambridge, 1989.

14. M. E. H. Ismail, J. Letessier, and G. Valent, Linear birth and death models and associated Laguerre polynomials, J. Approx. Theory 56 (1988), 337-348.

15. M. E. H. Ismail, J. Letessier, G. Valent, and J. Wimp, Associated Wilson polynomials, Canad. J. Math. 42 (1990), 659-695.

16. M. E. H. Ismail and D. Masson, Two families of orthogonal polynomials related to Jacobi polynomials, Rocky Mountain J. Math. 21 (1991) (to appear).

17. M. E. H. Ismail and J. A. Wilson, Asymptotic and generating relations for the q-Jacobi and ${ }_{4} \phi_{3}$ polynomials, J. Approx. Theory 36 (1982), 43-54.

18. W. Jones and W. Thron, Continued fractions, Cambridge Univ. Press, Cambridge, 1982.

19. D. Masson, The rotating harmonic oscillator eigenvalue problem. I. Continued fractions and analytic continuation, J. Math. Physics 24 (1983), 2074-2088.

20. _. Wilson polynomials and some continued fractions of Ramanujan, Rocky Mountain J. Math. 20 (1990) (to appear).

21. B. Nassrallah and M. Rahman, Projection formulas, a reproducing kernel and a generating function for $q$-Wilson polynomials, SIAM J. Math. Anal. 16 (1985), 186-197.

22. P. Nevai, Orthogonal polynomials, Mem. Amer. Math. Soc., no. 213, Providence, R.I., 1979.

23. F. W. J. Olver, Asymptotics and special functions, Academic Press, New York, 1974.

24. M. Rahman and A. Verma, A q-integral representation of Rogers' $q$-ultraspherical polynomials and some applications, Constr. Approx. 2 (1986), 1-10.

25. M. Rahman, q-Wilson function of the second kind, SIAM J. Math. Anal. 17 (1986), 12801286.

26. D. Sears, On the transformation theory of basic hypergeometric functions, Proc. London Math. Soc. (2) 53 (1951), 158-180.

27. J. A. Shohat and J. D. Tamarkin, The problem of moments, rev. ed., Math. Surveys, vol. 1, Amer. Math. Soc., Providence, R.I., 1950.

28. L. J. Slater, Generalized hypergeometric functions, Cambridge Univ. Press, Cambridge, 1966.

29. G. Szegö, Orthogonal polynomials, 4th ed., Colloq. Publ., vol. 23, Amer. Math. Soc., Providence, R.I., 1975.

30. J. Wilson, Hypergeometric series, recurrence relations and some orthogonal functions, Doctoral Dissertation, Univ. of Wisconsin, Madison, 1978. 31. $\frac{}{701}$, Some hypergeometric orthogonal polynomials, SIAM J. Math. Anal. 11 (1980), 690-

32. J. Wimp, Computations with recurrence relations, Pitman, London, 1984.

33. __ Explicit formulas for the associated Jacobi polynomials and some applications, Canad. J. Math. 39 (1987), 983-1000.

Department of Mathematics, University of South Florida, Tampa, Florida 33620

Department of Mathematics and Statistics, Carleton University, Ottawa, Ontario, Canada K1S 5B6 